\title{
Interleaving Isotactics - An Equivalence Notion on Behaviour Abstractions
}

\author{
Artem Polyvyanyy ${ }^{\mathrm{a}, 1, *}$, Jan Sümeli $^{\mathrm{b}}$, Matthias Weidlich ${ }^{\mathrm{c}}$ \\ ${ }^{a}$ The University of Melbourne, Australia \\ ${ }^{b}$ Technische Universität Berlin, Germany \\ ${ }^{c}$ Humboldt-Universität zu Berlin, Germany
}

\begin{abstract}
We study the equivalence of models that capture the behaviour of systems, such as process-oriented information systems. We focus on models that are not related by a bijection over their actions, but by an alignment between sets of their actions. For this setting, we propose interleaving isotactics as an equivalence notion based on abstractions that are induced by the alignment. We demonstrate that this notion is grounded in trace equivalence, provide a temporal logic characterisation of the properties it preserves, prove decidability of the respective verification problems, and present an implementation of a decision procedure for the equivalence notion.
\end{abstract}

Keywords: Behavioural equivalence, bisimulation, process semantics, behavioural abstraction, model matching

\section{Introduction}

The behaviour of a system is often described by a model that defines a set of actions and causal dependencies for their execution. Such models, also referred to as process models, have been widely adopted in the design and implementation of software systems in general [13] and process-oriented information systems in particular 22. Various applications require an assessment of the equivalence of two models that describe a system's behaviour. As examples, we consider applications from the domain of process-oriented systems:

Variation Management: In large organisations, a single process, and thus a single type of information system, exists in many variations due to country-specific legal requirements, deviations in the IT infrastructure, or organisational differences 32 . Models that describe these variations specify the same functionality, yet rely on different sets of actions as basic building blocks. Ensuring that the models are free of behavioural contradictions is a major concern when managing process variations, see 28 .

Implementation of Reference Models: For several domains, best-practices and well-established standard procedures have been published as reference models [10, 23. In order to make effective use of reference models, their equivalence needs to be assessed with respect to models that have been tailored and customised

\footnotetext{
${ }^{*}$ Corresponding author

Email addresses: artem.polyvyanyy@unimelb.edu.au (Artem Polyvyanyy), jan.suermeli@tu-berlin.de (Jan Sürmeli), matthias.weidlich@hu-berlin.de (Matthias Weidlich)

${ }^{1}$ This work was initiated at the Queensland University of Technology, Brisbane, Australia.
} 
Model $m_{1}$ :

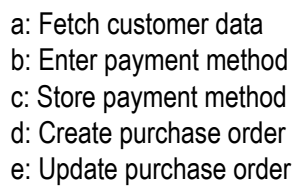

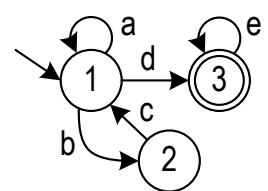

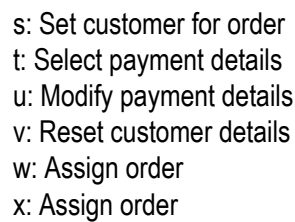

Model $\mathrm{m}_{2}$ :

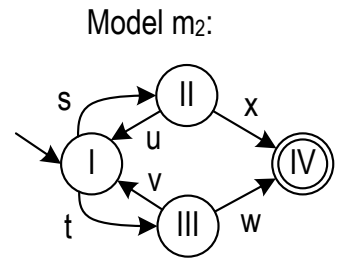

Alignment:

$\{a\} \bowtie\{s, v\}$

$\{b, c\} \bowtie\{t, u\}$

$\{d, e\} \bowtie\{s, w, x\}$

Figure 1. Two finite state machines modelling an excerpt of a business process and an alignment.

to a particular implementation environment. However, not all actions defined in a reference model may have a direct counterpart in the implementation.

Process Querying: Process querying studies methods for managing, e.g., retrieving or manipulating, repositories of models that describe observed and/or envisioned processes [17. It involves correlation models that relate actions of different abstractions of a system to each other (e.g., actions of a reference model and their corresponding implementations). Behavioural equivalences can be used to induce correlation models.

The above applications have in common that the models, for which equivalence shall be assessed, typically assume different levels of abstraction. In that case, the semantic correspondence between the actions of such models cannot be captured by a bijection. Rather, actions are grouped in either model and the groups of one model are related to the groups of another model by means of a binary relation, called alignment ${ }^{2}$ Alignments are typically constructed using techniques for automated model matching [4, 29. Note that the groups that are part of the alignment may overlap, i.e., a certain action can be interpreted in terms of different groups of actions in the other model. Hence, we do not narrow the scope to alignments that relate occurrences of actions to each other, see [20, since those are not discovered by matching techniques.

For illustration, we consider two finite state machines that depict a process to issue purchase orders, as implemented in two different systems, see Figure 1. Models $m_{1}$ and $m_{2}$ both contain actions related to the handling of customer data, the modification of payment details, as well as the actual order setup. Yet, when comparing the models, neither the actions nor their occurrences can be related by a bijection. For instance, the action ' $a$ : Fetch customer data' in model $m_{1}$ relates to two actions, ' $s$ : Set customer for order' and ' $v$ : Reset customer details' in model $m_{2}$. This relation cannot be traced back to established notions of hierarchical action refinements [5, 27. Action $s$ in model $m_{2}$ comprises functionality that does not only relate to the handling of customer data, but also includes the setup of the order. As such, it also relates to actions ' $d$ : Create purchase order' and ' $e$ : Update purchase order' in model $m_{1}$. These complex relations between the actions are captured in the alignment in Figure 1. The proposed alignment relates three sets of actions of either model to each other.

In this paper, we address the problem of assessing behavioural equivalence of two models in the context of a given alignment. We solve this problem by verifying equivalence based on abstractions, i.e., on the groups of actions induced by the alignment, rather than the individual actions.

Taking up the example in Figure 1, the alignment defines the abstractions for the verification of equivalence. The intuition of our approach is illustrated in Figure 2, where $r_{i}$ is an exemplary run of machine $m_{i}$, for $i \in\{1,2\}$. These runs induce traces $w_{1}$ and $w_{2}$ that contain sets of groups of actions, which can be seen as the interpretation of the runs under the given alignment. For instance, the occurrence of action $s$ in run $r_{2}$ is assigned all the groups of actions containing $s$ in the alignment definition, namely $\{s, v\}$ and $\{s, w, x\}$.

Based thereon, traces are compared by resolving each choice between groups of actions that have been assigned to action occurrences, while considering the maximal sequences of these groups. In the example, the first occurrence of action $s$ in run $r_{2}$ can be resolved as $\{s, v\}$ (setting and resetting customer data), which, under $\{a\} \bowtie\{s, v\}$, is in line with the occurrence of $a$ in $r_{1}$ (fetching customer data). Further, due to $\{b, c\} \bowtie\{t, u\}$, both occurrences of $b$ and $c$ in $r_{1}$ (entering and storing the payment method) correspond

\footnotetext{
${ }^{2}$ The term alignment used in this work is not to be confused with its use in process mining, where business alignment 24] refers to the practice of comparing the real behaviour of an information system or its users with the intended or expected behaviour and alignment refers to a concrete technique for quantifying differences between the observed and expected behaviour [25].
} 


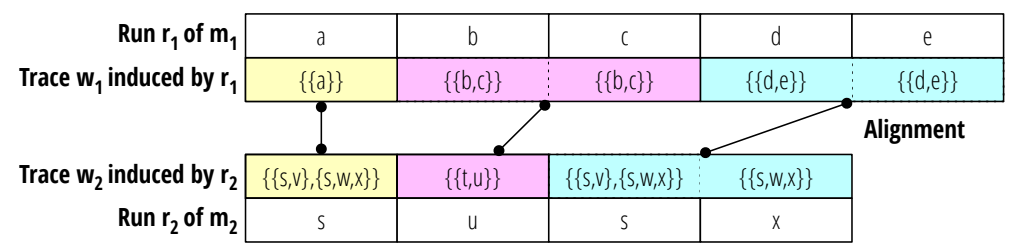

Figure 2. Two example runs of the state machines from Figure 1 and their traces induced by the alignment.

to the occurrence of $u$ in $r_{2}$ (modifying payment details). Finally, $\{d, e\} \bowtie\{s, w, x\}$ enables the abstraction of occurrences of $d$ and $e$ in $r_{1}$ (creating and updating a purchase order) and relating them to the second occurrence of $s$ followed by $x$ in $r_{2}$ (setting the customer and assigning an order). Here, unlike the first occurrence of $s$ that was resolved as $\{s, v\}$, the second occurrence is resolved as $\{s, w, x\}$.

This example illustrates that both runs $r_{1}$ and $r_{2}$ 'mirror' the behaviour of each other under the given alignment: After handling the customer data, the payment details are set, before the purchase order is managed. The equivalence verification relies on the explicit resolution of action occurrences when the action is part of more than one group in the alignment. All the runs of a system must be mirrored by runs of the other system to yield a behavioural equivalence of the systems.

Related Notions of Equivalence. Behavioural equivalences have been studied for decades, yet, established notions, see [3, 26, are not applicable for the setting outlined above as they impose the assumption of a bijection between the actions of two models. Thus, the traditional setting of equivalence verification can be seen as a special case of the setting addressed here, requiring that the alignment is of a particular structure.

As illustrated by the example, we target a setting where differences in the number of occurrences are abstracted. Such situations have been addressed by stutter behavioural equivalences, which are variants of standard behavioural equivalences that allow actions to be mimicked by sequences of actions (rather than by single actions) 6]. In this way, an action in an abstract model can be modelled as a sequence of actions in a concrete model [1. Stuttering equivalences are not applicable once the alignment relation defines overlapping groups of actions. As such, they are limited to alignments that are grounded in hierarchical refinements.

Recently, there have been several attempts to define behavioural equivalence for general alignments. Specific notions have been proposed for different semantics: interleaving, linear time semantics 30; interleaving, branching time semantics [31; and concurrent, linear time semantics 18. However, all these existing notions are ad-hoc, in the sense that:

(1) It is not known whether these notions are proper generalisations of the well-established behavioural equivalences. That is, if an alignment collapses to a bijection between the actions of two models, it is not known whether the proposed notions coincide with some known equivalences.

(2) It is not known what kind of system properties these notions preserve. That is, if two models show one of the proposed equivalences, it is not known whether there is a class of properties that is preserved, e.g., in terms of logic formulae as presented in 14 for standard equivalences and in 12 for stutter equivalences.

(3) It is not known, if and under which constraints any of the proposed notions is decidable.

Contributions. To assess behavioural equivalence of aligned models, we propose the notion of interleaving isotactics. This notion is inspired by earlier ideas on concurrent, linear time isotactics [18] that, however, at this stage suffers from the aforementioned three open issues. The intuition of isotactics (from the Greek:

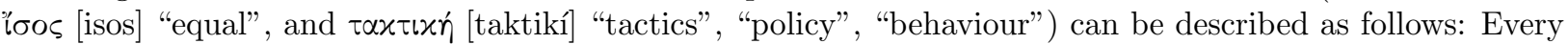
occurrence of an action can be mimicked by an arbitrary number of occurrences of any subset of actions of some aligned group of actions, regardless of the structural relations of these actions in the model. Indeed, it seems more natural to reason on the level of occurrences of actions rather than their structural relations in models when reasoning about behavioural equivalence.

Concretely, this paper contributes:

C1: A grounding of isotactics in an established behavioural equivalence. Trace equivalence and isotactics coincide for simple alignments (bijections over singleton sets of actions) and repetition-free sets of runs. 
C2: A Linear Temporal Logic (LTL) characterisation of the system properties preserved by isotactics. Given an alignment, we show that tactic-invariant LTL formulae are preserved, regardless of the actual systems.

C3: Decidability of the logic characterisation. Tactic-invariance of LTL-formulae is decidable.

C4: Decidability of the equivalence. Isotactics is decidable for finite state machines.

C5: An open-source implementation of a decision procedure for isotactics for finite state machines. This implementation is based on the arguments of the decidability proof and is publicly available 3

This paper proceeds with Section 2 that lists formal notions used to support the subsequent discussions. In Section 3, we formalise the alignment of (models of) systems and propose the notion of isotactics. The main results of this paper are summarised in Section 4. Sections 5 to 7 prove and discuss the main results. The paper closes with concluding remarks in Section 8. Detailed proofs of all the results of this paper can be found in Appendix A. Appendix B exemplifies the constructions used in one of the proofs of this paper.

\section{Formal Framework}

This section introduces formal notions that are used to support subsequent discussions.

Basic Notations. $\mathbb{N}$ denotes the set of all natural numbers including zero. Let $i, j \in \mathbb{N}$. By $\min (i, j)$ and $\max (i, j)$, we denote the minimum and maximum of $i$ and $j$, respectively. Let $A$ be a set. Then, by $\wp(A), \wp_{>0}(A)$, and $\wp_{=1}(A)$, we denote the power set of $A, \wp(A) \backslash\{\varnothing\}$, and $\left\{x \in \wp_{>0}(A) \mid \exists a \in A: x=\{a\}\right\}$, respectively. Let $B$ be a set, and $f: A \rightarrow B$ be a function. If $A^{\prime} \subseteq A$, then by $f\left(A^{\prime}\right)$ we denote the set $\{b \in B \mid$ $\left.\exists a \in A^{\prime}: f(a)=b\right\}$. Let $R$ be a binary relation between $A$ and $B$. Then, $R^{-1}:=\{(b, a) \in B \times A \mid(a, b) \in R\}$ denotes the inverse of $R$. Let $\equiv$ be an equivalence relation on $A$. Then, by $\langle a\rangle_{\equiv}$, where $a \in A$, we denote the equivalence class of $A$ by $\equiv$ that contains $a$. Moreover, by $A / \equiv$ we denote the set of all equivalence classes of $A$ by $\equiv$, i.e., $A / \equiv:=\left\{x \in \wp_{>0}(A) \mid \exists a \in A:\langle a\rangle_{\equiv}=x\right\}$. We write $A^{*}$ to denote the set of all (finite) words over $A$, including the empty word $\varepsilon$. Let $w:=a_{1} \ldots a_{n} \in A^{*}$ be a word. Then, $w(k):=a_{k}$, where $k \in\{1, \ldots, n\}$, denotes the $k$-th character of $w$, and $|w|:=n$ denotes the length of $w$. Let $k \in\{1, \ldots,|w|\}$, then $w[k\rangle$ denotes the suffix $w(k) \ldots w(n)$ of $w$ starting from the $k$-th character of $w$. We call $w$ repetition-free iff for all $k \in\{1, \ldots, n-1\}: w(k) \neq w(k+1)$. We call a set $W$ of words repetition-free, if each of its members $w \in W$ is repetition-free. The concatenation of two words $w=a_{1} \ldots a_{n}$ and $w^{\prime}=a_{1}^{\prime} \ldots a_{m}^{\prime}$ is defined as $w w^{\prime}:=a_{1} \ldots a_{n} a_{1}^{\prime} \ldots a_{m}^{\prime}$. For example, $\hat{w}=$ ababahalamaha is a repetition-free word that results from the concatenation of $w=a b a b a$ and $w^{\prime}=$ halamaha, i.e., $\hat{w}=w w^{\prime}$.

Traces and Linear Temporal Logic. Let $\kappa$ be a finite set. A $\kappa$-trace is an element of $\wp(\kappa)^{*}$, i.e., a finite sequence of subsets of $\kappa$. For $i \in\{1,2\}$, let $\kappa_{i}$ be a finite set, and let $W_{i}$ be a set of $\kappa_{i}$-traces. $W_{1}$ and $W_{2}$ are trace equivalent up to a bijection $b$ from $\kappa_{1}$ to $\kappa_{2}$, iff $b$ induces an isomorphism between traces in $W_{1}$ and $W_{2}$.

Definition 2.1 (Trace Equivalence). For $i \in\{1,2\}$, let $\kappa_{i}$ be a finite set, and let $W_{i}$ be a set of $\kappa_{i}$-traces. Let $b$ be a bijection from $\kappa_{1}$ to $\kappa_{2}$. Then, $W_{1}$ and $W_{2}$ are trace equivalent up to $b$ iff there exists a bijection $R$ from $W_{1}$ to $W_{2}$ such that for all $w_{1} \in W_{1}$ it holds that (i) $\left|w_{1}\right|=\left|R\left(w_{1}\right)\right|$ and (ii) for all $i \in\left\{1, \ldots,\left|w_{1}\right|\right\}$ it holds that $b\left(w_{1}(i)\right)=R\left(w_{1}\right)(i)$.

By $\operatorname{LTL}[\kappa]$, we denote the set of all LTL-Formulae [1, 16] (without the next operator) over a set $\kappa$ that are given by the following expression:

$$
\varphi::=\text { true }|K| \neg \psi\left|\psi_{1} \vee \psi_{2}\right| \psi_{1} \mathrm{U} \psi_{2} \text {, where } K \in \kappa \text { and } \psi, \psi_{1}, \psi_{2} \in \operatorname{LTL}[\kappa] \text {. }
$$

Let $\varphi \in \operatorname{LTL}[\kappa]$ and $w$ be a $\kappa$-trace. Then, $w$ satisfies $\varphi$, written $w \vDash \varphi$, iff $\varphi=$ true or:

- $\varphi=K, K \in \kappa$, and $K \in w(1)$,

- $\varphi=\neg \psi$, and $w \neq \psi$,

- $\varphi=\psi_{1} \vee \psi_{2}$, and there exists $i \in\{1,2\}: w \vDash \psi_{i}$, or

- $\varphi=\psi_{1} \mathrm{U} \psi_{2}$, and there exists $k \in\{1, \ldots,|w|\}$ with $w[k\rangle \vDash \psi_{2}$ and $w[i\rangle \vDash \psi_{1}$ for all $i \in\{1, \ldots, k-1\}$.

$\sqrt[3]{\text { https://github.com/Isotactics/deciding-isotactics }}$ 
Other Boolean connectives, e.g., $\wedge, \Rightarrow$, and $\Leftrightarrow$, can be obtained from $\neg$ and $\vee$ in the standard way. As usual, the until operator $(\mathrm{U})$ can be used to define temporal modalities $\mathrm{F}$ (eventually) and $\mathrm{G}$ (globally). In particular, $\mathrm{F} \psi:=$ true $\mathrm{U} \psi$ and $\mathrm{G} \psi:=\neg \mathrm{F} \neg \psi$.

Finite State Machines. We rely on a common notion of finite state machines [9, 19]:

Definition 2.2 (Finite State Machine). A finite state machine (FSM) is a 5-tuple $S:=\left(\mathcal{Q}, \Lambda, \rightarrow, q^{i n i}, F\right)$, where $\mathcal{Q}$ is a finite set of states, $\Lambda$ is a set of labels, $\mathcal{Q}$ and $\Lambda$ are disjoint, $\rightarrow \subseteq \mathcal{Q} \times \Lambda \times \mathcal{Q}$ is the transition relation, $q^{\text {ini }} \in \mathcal{Q}$ is the initial state, and $F \subseteq \mathcal{Q}$ is the set of final states.

We say that $S$ is defined over $\Lambda$. By $p \stackrel{\lambda}{\rightarrow} q$, where $p, q \in \mathcal{Q}$ and $\lambda \in \Lambda$, we denote the fact that $(p, \lambda, q) \in \rightarrow$. We say that $S$ accepts a word $w \in \Lambda^{*}$, iff (i) $w$ is the empty word, i.e., $w=\varepsilon$, and $q^{i n i} \in F$, or (ii) there exists a sequence $\rho \in \mathcal{Q}^{*}$ of states of length $|w|+1$, such that $\rho(1)=q^{i n i}, \rho(|w|+1) \in F$, and for all $i \in\{1, \ldots,|w|\}$ it holds that $\rho(i) \stackrel{w(i)}{\longrightarrow} \rho(i+1)$; we refer to a word accepted by $S$ as a run of $S$.

By $\mathcal{L}(S)$, we denote the set of all runs of $S$, i.e., the language of $S$. Two FSMs $S_{1}$ and $S_{2}$ are languageequivalent iff $\mathcal{L}\left(S_{1}\right)=\mathcal{L}\left(S_{2}\right)$. Two FSMs are illustrated in Figure 1, using common notation. For instance, FSM $m_{1}:=\left(\mathcal{Q}, \Lambda, \rightarrow, q^{i n i}, F\right)$ has three states, $\mathcal{Q}=\{1,2,3\}$, with the initial state $q^{i n i}=1$ and the set of final states $F=\{3\}$. Its set of labels is $\Lambda=\{a, b, c, d, e\}$ and its transition relation is defined by $1 \stackrel{a}{\rightarrow} 1,1 \stackrel{b}{\rightarrow} 2,2 \stackrel{c}{\rightarrow} 1$, $1 \stackrel{d}{\rightarrow} 3$, and $3 \stackrel{e}{\rightarrow} 3$.

\section{Equivalence of Aligned Systems}

We consider the behaviour of a system in terms of interleaving, linear time semantics, i.e., as a set of runs, where a run is a finite sequence over a set of labels. A run is produced by a system model, such as an FSM, Petri net, or Turing machine. Against the background of describing the behaviour of systems, a label often represents an action. However, other interpretations are possible: A label could represent a state, a state predicate, a configuration, or a similar concept. The basis for aligning system models comprises:

1. Grouping of labels. Labels that shall be considered jointly when comparing the behaviour of systems are part of a single group. The groups may overlap, i.e., a label may be a member of more than one group. Intuitively, this corresponds to different interpretations of a single label. For instance, in Figure 1, the action 's: Set customer for order' can be interpreted together with action ' $v$ : Reset customer details' as the sheer retrieval of customer data, whereas with actions ' $w, x$ : Assign order', it relates to processing of a purchase order. One can 'eliminate' labels by not assigning them to any group. The respective labels are then not further considered in the verification of equivalence.

2. Relating the groups of one model with the groups of another model by means of a binary relation, called alignment. The alignment thereby defines a structural relation between groups of labels for which occurrences shall be considered as equivalent. Referring to Figure 1, as detailed above already, the functionality of action ' $a$ : Fetch customer data' in model $m_{1}$ is reflected in the functionality behind two actions, 's: Set customer for order' and 'v: Reset customer details' in model $m_{2}$.

Based thereon, one can assess behavioural equivalence of two models by:

1. Abstracting each model based on the groups. The idea here is that the behaviour of a system is no longer considered in terms of runs over a set of labels, but in terms of runs over groups. For two runs of our running example, this abstraction is illustrated in Figure 2, in terms of the traces $w_{1}$ and $w_{2}$ that are induced by the alignment.

2. Verifying whether, according to the alignment, the abstract models are behaviourally equivalent. That is, it is checked whether two systems show the same behaviour once the latter is interpreted in terms of runs over groups. For the example in Figure 2, we see that indeed, both runs describe the same behaviour of first handling customer data, which is followed by the setup of payment details, before the management of the purchase order.

Based on the above intuition, this section develops the formalisation of the proposed equivalence notion: We first define groupings and alignments (Section 3.1). Based thereon, we clarify the notions needed to abstract a model based on groups (Section 3.2), which are then used to verify equivalence (Section 3.3). 


\begin{tabular}{|c|c|c|c|c|c|}
\hline \multirow{4}{*}{$\begin{array}{r}\text { Run } r_{1} \text { of } m_{1} \\
\text { Trace } w_{1} \text { induced by } r_{1} \\
\text { Tactic } \theta_{1} \text { of } w_{1} \\
\{1,2,3,4,5\} / \theta_{1}\end{array}$} & a & $b$ & c & $d$ & \multirow{2}{*}{$\frac{e}{\{\{d, e\}\}}$} \\
\hline & $\{\{a\}\}$ & $\{\{b, c\}\}$ & $\{\{b, c\}\}$ & $\{\{d, e\}\}$ & \\
\hline & $\{\{a\}\}$ & $\{\{b, c\}\}$ & $\{\{b, c\}\}$ & $\{\{d, e\}\}$ & $\{\{d, e\}\}$ \\
\hline & $\{1\}$ & \multicolumn{2}{|c|}{2,3} & \multicolumn{2}{|c|}{4,5} \\
\hline & & & & & \\
\hline$\{1,2,3,4\} / \theta_{2}$ & $\{1\}$ & $\{2\}$ & \multicolumn{2}{|c|}{3,4} & \\
\hline Tactic $\theta_{2}$ of $r_{2}$ & $\{\{s, v\}\}$ & $\{\{t, u\}\}$ & $\{\{S, W, X\}\}$ & $\{\{S, W, X\}\}$ & \\
\hline Trace $w_{2}$ induced by $r_{2}$ & $\{\{S, V\},\{S, W, X\}\}$ & $\{\{t, u\}\}$ & $\{\{s, V\},\{s, w, X\}\}$ & $\{\{S, W, X\}\}$ & \\
\hline Run $r_{2}$ of $m_{2}$ & s & u & s & $x$ & \\
\hline
\end{tabular}

Figure 3. The main concepts illustrated using the runs from Figure 2 For $i \in\{1,2\}, r_{i}$ is a run of $m_{i}$ from Figure 1 is the trace of $r_{i}$, and $\theta_{i}$ is a tactic of $w_{i}$. The traces $w_{1}$ and $w_{2}$ are aligned by tactics $\theta_{1}$ and $\theta_{2}$ because $\{a\} \bowtie\{s, v\},\{b, c\} \bowtie\{t, u\}$ and $\{d, e\} \bowtie\{s, w, x\}$. Another tactic of $w_{2}$ is $\theta_{2}^{\prime}=\{\{s, v\}\}\{\{t, u\}\}\{\{s, v\}\}\{\{s, w, x\}\}$. However, $w_{1}$ and $w_{2}$ are not aligned by $\theta_{1}$ and $\theta_{2}^{\prime}$, because the second $\{\{s, v\}\}$ in $\theta_{2}^{\prime}$ does not have a counterpart in $\theta_{1}$. In fact, $\theta_{1}$ and $\theta_{2}$ is the only combination of tactics aligning $w_{1}$ and $w_{2}$. Hence, to align $w_{1}$ and $w_{2}$, the action $s$ has to be resolved once as $\{\{s, v\}\}$ and once as $\{\{s, w, x\}\}$.

Finally, we elaborate on how behavioural properties of systems, defined in LTL, shall be interpreted in the context of an alignment Section 3.4.

\subsection{Groupings and Alignments}

This section defines a grouping of a set of labels and an alignment between sets of labels. Intuitively, a grouping is a collection of sets of labels, in which every set encodes a group of actions of a system that shall be considered together in some behavioural analysis. An alignment specifies correspondences between groups of actions of two systems, thereby establishing which groups of labels relate to the same functionality described by the system models.

Definition 3.1 (Grouping). A grouping of a set of labels $\Lambda$ is a set $\kappa \subseteq \wp_{>0}(\Lambda)$. A set $K \in \kappa$ is a $\kappa$-group of $\Lambda$. Referring to the labels of the FSMs in Figure 1. e.g., $\gamma_{1}:=\{\{a\},\{b, c\},\{d, e\}\}$ and $\gamma_{2}:=\{\{s, v\},\{t, u\},\{s, w, x\}\}$ are groupings of the sets of labels $\Gamma_{1}:=\{a, b, c, d, e\}$ and $\Gamma_{2}:=\{s, t, u, v, w, x\}$, respectively. Given a grouping $\kappa$ of $\Lambda$ and a label $\lambda \in \Lambda$, by $\mathcal{G}_{\kappa}(\lambda)$ we denote the set of all $\kappa$-groups that contain $\lambda$, i.e., $\mathcal{G}_{\kappa}(\lambda):=\{K \in \kappa \mid \lambda \in K\}$. For instance, it holds that $\mathcal{G}_{\gamma_{2}}(s)=\{\{s, v\},\{s, w, x\}\}$, which in the example relates to the different interpretations of action ' $s$ : Set customer for order', either in the context of retrieving the customer data or related to the processing of a purchase order.

Definition 3.2 (Alignment). For $i \in\{1,2\}$, let $\Lambda_{i}$ be a set of labels, and let $\kappa_{i} \subseteq \wp_{>0}\left(\Lambda_{i}\right)$ be a grouping of $\Lambda_{i}$. Then, an alignment between $\Lambda_{1}$ and $\Lambda_{2}$ is a relation $\bowtie \subseteq \kappa_{1} \times \kappa_{2}$, written $\bowtie: \Lambda_{1} \otimes \Lambda_{2}$, which relates the $\kappa_{1}$-groups of $\Lambda_{1}$ with the $\kappa_{2}$-groups of $\Lambda_{2}$.

Every alignment $\bowtie$ induces groupings $\stackrel{i}{\bowtie}:=\left\{K_{i} \in \wp_{>0}\left(\Lambda_{i}\right) \mid K_{1} \bowtie K_{2}\right\}$ of $\Lambda_{i}, i \in\{1,2\}$.

For example, $\{(\{a\},\{s, v\}),(\{b, c\},\{t, u\}),(\{d, e\},\{s, w, x\})\} \subseteq \gamma_{1} \times \gamma_{2}$ is an alignment between $\Gamma_{1}$ and $\Gamma_{2}$ used in Figure 1, it induces groupings $\gamma_{1}$ and $\gamma_{2}$ proposed above. As mentioned above, this alignment specifies that action $a$ in model $m_{1}$ corresponds to actions $s$ and $v$ in model $m_{2}$. Furthermore, according to this alignment, actions $b$ and $c$ correspond to actions $t$ and $u$, and actions $d$ and $e$ correspond to actions $s, w$, and $x$.

\subsection{Comparing Runs based on Tactics}

This section proposes a method for comparing runs of systems based on their tactics induced by alignments. In the remainder of this section, we will illustrate the essential concepts using the initial example of the state machines in Figure 1 and their runs depicted in Figure 2, This example is extended in Figure 3, which also points to the respective definitions in this section.

Let $\Lambda$ be a set of labels, and let $\kappa$ be a grouping of $\Lambda$. Every run $\sigma \in \Lambda^{*}$ induces the $\kappa$-trace $w:=$ $\mathcal{G}_{\kappa}(\sigma(1)) \ldots \mathcal{G}_{\kappa}(\sigma(|\sigma|))$. Then, the $\kappa$-induced trace of $\sigma$ is a sequence of sets of $\kappa$-groups obtained from $w$ by removing all its elements that are equal to $\varnothing$ without changing the order of the remaining elements. 
Definition 3.3 (Induced Trace). Let $\Lambda$ be a set of labels, let $\kappa$ be a grouping of $\Lambda$, and let $\mathcal{T}_{\kappa}: \Lambda^{*} \rightarrow \wp_{>0}(\kappa)^{*}$ be given by:

- $\mathcal{T}_{\kappa}(\varepsilon):=\varepsilon$.

- Let $\sigma \in \Lambda^{*}, \lambda \in \Lambda$. Then, $\mathcal{T}_{\kappa}(\sigma \lambda):= \begin{cases}\mathcal{T}_{\kappa}(\sigma) \mathcal{G}_{\kappa}(\lambda) & \text { if } \mathcal{G}_{\kappa}(\lambda) \neq \varnothing, \\ \mathcal{T}_{\kappa}(\sigma) & \text { otherwise. }\end{cases}$

Given a run $\sigma \in \Lambda^{*}, \mathcal{T}_{\kappa}(\sigma)$ is the $\kappa$-induced trace of $\sigma$.

As an example, we consider groupings of labels $\gamma_{1}:=\{\{a\},\{b, c\},\{d, e\}\}$ and $\gamma_{2}:=\{\{s, v\},\{t, u\},\{s, w, x\}\}$ as defined in Section 3.1. Also, let $r_{1}:=a b c d e$ be a run of model $m_{1}$, which describes that after fetching some customer data, a payment method is entered and stored, after which a purchase order is created and updated. Run $r_{2}:=$ susx of model $m_{2}$, in turn, describes a situation in which a customer of an order is set initially, after which the payment details are modified, so that the customer data of the order needs to be set again, before the order is eventually assigned. Then, $\{\{a\}\}\{\{b, c\}\}\{\{b, c\}\}\{\{d, e\}\}\{\{d, e\}\}$ is the $\gamma_{1}$-induced trace of $r_{1}$, while $\{\{s, v\},\{s, w, x\}\}\{\{t, u\}\}\{\{s, v\},\{s, w, x\}\}\{\{s, w, x\}\}$ is the $\gamma_{2}$-induced trace of $r_{2}$, see Figure 3

We compare traces based on tactics. Intuitively, a tactic represents a specific interpretation of the run of a system in terms of specific groups. While an induced trace describes all possible interpretations of a run, a tactic requires to choose among them. Let $\kappa$ be a grouping of a set of labels $\Lambda$, and let $w \in \wp_{>0}(\kappa)^{*}$ be a $\kappa$-trace. A tactic $\theta$ of $w$ selects $K \in w(i)$ for each position $i$ of $w$.

Definition 3.4 (Tactic). A tactic of a $\kappa$-trace $w \in \wp_{>0}(\kappa)^{*}$, where $\kappa$ is a finite set, is a $\kappa$-trace $\theta \in \wp_{=1}(\kappa)^{*}$ such that $|\theta|=|w|$ and $\theta(i) \subseteq w(i)$ for all $i \in\{1, \ldots,|w|\}$.

Continuing with our running example, the $\gamma_{1}$-induced trace $\mathcal{T}_{\gamma_{1}}$ (abcde) has only a single tactic, which is illustrated in Figure 3 The $\gamma_{2}$-induced trace $\mathcal{T}_{\gamma_{2}}(\operatorname{sus} x)$, in turn, has four tactics:

- $\theta_{2}^{\prime}:=\{\{s, v\}\}\{\{t, u\}\}\{\{s, v\}\}\{\{s, w, x\}\}$,

- $\theta_{2}:=\{\{s, v\}\}\{\{t, u\}\}\{\{s, w, x\}\}\{\{s, w, x\}\}$,

- $\theta_{2}^{\prime \prime}:=\{\{s, w, x\}\}\{\{t, u\}\}\{\{s, v\}\}\{\{s, w, x\}\}$, and

- $\theta_{2}^{\prime \prime \prime}:=\{\{s, w, x\}\}\{\{t, u\}\}\{\{s, w, x\}\}\{\{s, w, x\}\}$.

The above tactics essentially stem from the different means to interpret the action 's: Set customer for order' in the original run, and their combinations. Tactic $\theta_{2}:=\{\{s, v\}\}\{\{t, u\}\}\{\{s, w, x\}\}\{\{s, w, x\}\}$ is illustrated in Figure 3

Each tactic $\theta$ induces an equivalence relation $=_{\theta}$ and a strict partial order $<_{\theta}$ on the set $\{1, \ldots,|\theta|\}$ of indices of $\theta$. Two indices are equivalent, if $\theta$ selects the same $K \in \kappa$ for them and all indices between them. Two indices are ordered if they are ordered inside $\theta$ but are not equivalent.

Definition 3.5 (Tactic-induced Relations). Let $\theta$ be a tactic of a $\kappa$-trace $w \in \wp_{>0}(\kappa)^{*}$, where $\kappa$ is a finite set. Then, ${ }_{\theta} \subseteq\{1, \ldots,|\theta|\} \times\{1, \ldots,|\theta|\}$ and $<_{\theta} \subseteq\{1, \ldots,|\theta|\} \times\{1, \ldots,|\theta|\}$ are defined as follows:

1. $i={ }_{\theta} j$ iff for all $k, l \in\{1, \ldots,|\theta|\}$ such that $\min (i, j) \leq k, \ell \leq \max (i, j)$ it holds that $\theta(k)=\theta(\ell)$.

2. $i<_{\theta} j$ iff $i<j$ and $i \neq_{\theta} j$.

We note that $=_{\theta}$ is an equivalence relation. We abbreviate $\langle i\rangle_{\theta_{\theta}}$ and $\{1, \ldots,|\theta|\} /=_{\theta}$ as $\langle i\rangle_{\theta}$ and $\{1, \ldots,|\theta|\} /{ }_{\theta}$, respectively. Let $i, j \in\{1, \ldots,|\theta|\}$, such that $i<_{\theta} j$. Then, for all $i^{\prime} \in\langle i\rangle_{\theta}$ and $j^{\prime} \in\langle j\rangle_{\theta}$, it holds that $i^{\prime}<_{\theta} j^{\prime}$. Hence, one can lift $<_{\theta}$ from $\{1, \ldots,|\theta|\}$ to $\{1, \ldots,|\theta|\} /\left.\right|_{\theta}$ : For all $i, j \in\{1, \ldots,|\theta|\},\langle i\rangle_{\theta}<_{\theta}\langle j\rangle_{\theta}$ iff $i<_{\theta} j$.

For example, the tactic $\theta_{2}:=\{\{s, v\}\}\{\{t, u\}\}\{\{s, w, x\}\}\{\{s, w, x\}\}$ induces the equivalence relation $=_{\theta_{2}}$ given by $\{(1,1),(2,2),(3,3),(4,4),(3,4),(4,3)\} ;=\theta_{2}$ induces three equivalence classes: $\{1\},\{2\}$, and $\{3,4\}$. Moreover, $<_{\theta_{2}}$ is given by $\{(1,2),(1,3),(1,4),(2,3),(2,4)\}$. Thus, it holds that $\{1\}<_{\theta_{2}}\{2\}<_{\theta_{2}}\{3,4\}$.

Let $\bowtie: \Lambda_{1} \otimes \Lambda_{2}$ be an alignment. For $i \in\{1,2\}$, let $w_{i} \in \wp(\dot{\star})^{*}$. Intuitively, $w_{1}$ and $w_{2}$ are aligned if there exist tactics $\theta_{1}$ and $\theta_{2}$ of $w_{1}$ and $w_{2}$, respectively, that can be aligned.

Definition 3.6 (Alignment of Tactics). Let $\bowtie: \Lambda_{1} \otimes \Lambda_{2}$ be an alignment. For $i \in\{1,2\}$, let $w_{i} \in \wp(\dot{\star})^{*}$, and let $\theta_{i}$ be a tactic of $w_{i}$. Tactics $\theta_{1}$ and $\theta_{2}$ are aligned by $\bowtie$, denoted by $\theta_{1} \bowtie \theta_{2}$, iff there exists a bijection $b$ from $\left\{1, \ldots,\left|\theta_{1}\right|\right\} / \theta_{1}$ to $\left\{1, \ldots,\left|\theta_{2}\right|\right\} / \theta_{2}$ such that: 
1. for all $i_{1} \in\left\{1, \ldots,\left|\theta_{1}\right|\right\}$ and $i_{2} \in\left\{1, \ldots,\left|\theta_{2}\right|\right\}$ it holds that $b\left(\left\langle i_{1}\right\rangle_{\theta_{1}}\right)=\left\langle i_{2}\right\rangle_{\theta_{2}}$ implies $\theta_{1}\left(i_{1}\right) \bowtie \theta_{2}\left(i_{2}\right)$, and

2. for all $i, j \in\left\{1, \ldots,\left|\theta_{1}\right|\right\}$ it holds that $\langle i\rangle_{\theta_{1}}<_{\theta_{1}}\langle j\rangle_{\theta_{1}}$ implies $b\left(\langle i\rangle_{\theta_{1}}\right)<_{\theta_{2}} b\left(\langle j\rangle_{\theta_{1}}\right)$.

We say that $w_{1}$ and $w_{2}$ are aligned by $\theta_{1}$ and $\theta_{2}$ w.r.t. $\bowtie$, and denote this by $\left(\theta_{1}, \theta_{2}\right): w_{1} \bowtie w_{2}$.

To illustrate the alignment of tactics, we again refer to Figure 3 . Here, the $\gamma_{1}$-induced trace $\mathcal{T}_{\gamma_{1}}(a b c d e)$ and the $\gamma_{2}$-induced trace $\mathcal{T}_{\gamma_{2}}($ sus $x)$ are aligned by tactics $\theta_{1}:=\{\{a\}\}\{\{b, c\}\}\{\{b, c\}\}\{\{d, e\}\}\{\{d, e\}\}$ and $\theta_{2}:=\{\{s, v\}\}\{\{t, u\}\}\{\{s, w, x\}\}\{\{s, w, x\}\}$ w.r.t. the alignment from Figure 1 one can construct bijection $b:=\{\{1\} \mapsto\{1\},\{2,3\} \mapsto\{2\},\{4,5\} \mapsto\{3,4\}\}$ from $\left\{1, \ldots,\left|\theta_{1}\right|\right\} / \theta_{1}$ to $\left\{1, \ldots,\left|\theta_{2}\right|\right\} / \theta_{2}$. This bijection formally encodes the intuition given earlier: In either system, customer data is handled first, before payment details are set, which is followed by the management of the purchase order.

In contrast to the above example, $\mathcal{T}_{\gamma_{1}}$ (ade) $=\{\{a\}\}\{\{d, e\}\}\{\{d, e\}\}$ and $\mathcal{T}_{\gamma_{2}}($ sus $x)$ cannot be aligned. Run ade represents the situation that handling of customer data is immediately followed by the management of the purchase order. Unlike run susx, run ade thus does not include any actions related to the setup of the payment details. Finally, illustrating that alignments abstract from cardinalities, for every $n \in \mathbb{N}$ it holds that $\mathcal{T}_{\gamma_{1}}(\underbrace{b c \ldots b c}_{n \text { times }} d)$ and $\mathcal{T}_{\gamma_{2}}(t w)$ can be aligned. Intuitively, this means that entering and storing the payment method repeatedly according to model $m_{1}$ is equivalent to a single selection of payment details in model $m_{2}$.

\subsection{Tactic Coverage and Isotactics}

We propose to compare two collections of traces based on tactic coverage and isotactics relations. The idea is that a collection of traces is "covered" by another collection of traces if for every trace in this collection one can find a trace in the other collection such that the tactics of these two traces are aligned.

Definition 3.7 (Tactic Coverage and Isotactics).

Let $\bowtie: \Lambda_{1} \otimes \Lambda_{2}$ be an alignment. For $i \in\{1,2\}$, let $W_{i}$ be a set of $\dot{x}$-traces.

1. $W_{1}$ and $W_{2}$ are in the (interleaving) tactic coverage relation w.r.t. $\bowtie$, denoted by $W_{1} \leqslant{ }_{\infty} W_{2}$, iff for every trace $w_{1} \in W_{1}$ there exists a trace $w_{2} \in W_{2}$ such that there exist tactics $\theta_{1}$ and $\theta_{2}$ of $w_{1}$ and $w_{2}$, respectively, such that $\left(\theta_{1}, \theta_{2}\right): w_{1} \bowtie w_{2}$.

2. $W_{1}$ and $W_{2}$ are in the (interleaving) isotactics relation w.r.t. $\bowtie$, denoted by $W_{1} \doteqdot_{\infty} W_{2}$, iff $W_{1} \aleph_{\infty} W_{2}$ and $W_{2} \aleph_{\mathbb{N}^{-1}} W_{1}$.

One can compare runs based on their induced traces, i.e., based on their abstractions induced by the alignment. For $i \in\{1,2\}$, let $\Lambda_{i}$ be a set of labels, let $\Sigma_{i} \subseteq \Lambda_{i}^{*}$ be a set of runs, and let $\bowtie: \Lambda_{1} \otimes \Lambda_{2}$ be an alignment. We

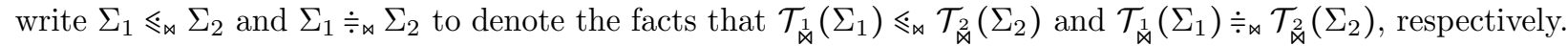

For our running example in Figure 1. one can verify that for every $\gamma_{1}$-induced trace $w_{1}$ of $m_{1}$, there exists some $\gamma_{2}$-induced trace $w_{2}$ of $m_{2}$ such that some tactics of $w_{1}$ and $w_{2}$ are aligned, and vice versa; this fact is justified in Section 7. Note that groupings $\gamma_{1}$ and $\gamma_{2}$ are proposed in Section 3.1 and are the groupings used to define alignment $\bowtie$ in Figure 1. Hence, it holds that $\mathcal{L}\left(m_{1}\right) \dot{\varpi}_{\bowtie} \mathcal{L}\left(m_{2}\right)$ and we say that $m_{1}$ and $m_{2}$ are isotactic w.r.t. $\bowtie$.

\subsection{Alignments and LTL}

To later explore the class of properties preserved by isotactics, we first need to review how such properties shall be interpreted in the presence of groupings and alignments. Considering properties formalised in LTL, there is a class of formulae that is of particular interest: Those for which the choice of tactics does not affect its truth value, called tactic-invariant LTL formulae. Below, we provide a formal characterisation of these formulae and how they are interpreted in the context of an alignment. This provides the basis to later show that tactic-invariant LTL formulae are indeed preserved by isotactics.

Let $\Lambda$ be a set of labels, let $\kappa \subseteq \wp_{>0}(\Lambda)$ be a grouping, let $\Sigma \subseteq \Lambda^{*}$, let $\sigma \in \Sigma$, and let $\varphi \in \operatorname{LTL}[\kappa]$. Then, $\sigma$ satisfies $\varphi$ w.r.t. $\kappa$, denoted by $(\sigma, \kappa) \vDash \varphi$, iff $\mathcal{T}_{\kappa}(\sigma) \vDash \varphi$. For example, given run $\sigma:=t v s x$ of model $m_{2}$ in Figure 1, it holds that $\{\{t, u\}\}\{\{s, v\}\}\{\{s, v\},\{s, w, x\}\}\{\{s, w, x\}\}$ is the $\gamma_{2}$-induced trace of $\sigma$, where $\gamma_{2}$ is defined in Section 3.1. Then, for sample formula $\varphi:=(\{s, v\} \vee\{t, u\}) \cup\{s, w, x\}$, we have $\left(\sigma, \gamma_{2}\right) \vDash \varphi$. Similarly, $\Sigma$ satisfies $\varphi$ w.r.t. $\kappa$, denoted by $(\Sigma, \kappa) \vDash \varphi$, iff for every $\sigma \in \Sigma$ it holds that $(\sigma, \kappa) \vDash \varphi$. 
Each tactic $\theta$ of a $\kappa$-trace $w$ is a $\kappa$-trace. Thus, every formula $\varphi \in \operatorname{LTL}[\kappa]$ can be evaluated over both $w$ and $\theta$. We call formula $\varphi \in \operatorname{LTL}[\kappa]$ tactic-invariant, if for every trace $w$ it holds that the truth value of $\varphi$ over $w$ and over every tactic $\theta$ of $w$ is the same.

Definition 3.8 (Tactic-Invariant LTL-Formula). Let $\Lambda$ be a set of labels and let $\kappa \subseteq \wp_{>0}(\Lambda)$ be a grouping of $\Lambda$. An LTL-formula $\varphi \in \operatorname{LTL}[\kappa]$ is tactic-invariant w.r.t. $\Lambda$ and $\kappa$, iff for every $w \in \mathcal{T}_{\kappa}\left(\Lambda^{*}\right)$ and every tactic $\theta$ of $w$ it holds that $w \vDash \varphi$ iff $\theta \vDash \varphi$.

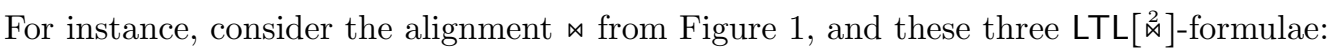

$$
\varphi_{1}:=\{t, u\} \cup(\{s, v\} \vee\{s, w, x\}), \quad \varphi_{2}:=\neg\{s, v\} \vee \neg\{s, w, x\}, \quad \text { and } \quad \varphi_{3}:=\neg \varphi_{2} .
$$

Clearly, $\varphi_{1}$ is tactic-invariant: The satisfying 2 -induced traces are of the form $w_{1} w_{2} w_{3}$ where $w_{1}$ is an element in $\{\{\{t, u\}\}\}^{*}$, e.g., $w_{1}=\varepsilon$ or $w_{1}=\{\{t, u\}\}\{\{t, u\}\}, w_{2}$ is a non-empty subset of $\{\{s, v\},\{s, w, x\}\}$, and $w_{3}$ is an arbitrary $\mathscr{x}^{2}$-induced trace. The tactics of such a trace $w_{1} w_{2} w_{3}$ are of the form $\theta_{1} \theta_{2} \theta_{3}$, where $\theta_{1}=w_{1}$ (because $w_{1}$ is a sequence of singleton sets), $\theta_{2}$ is either $\{\{s, v\}\}$ or $\{\{s, w, x\}\}$, and $\theta_{3}$ is any tactic of $w_{3}$. Because $\theta_{1}$ is a sequence of $\{\{t, u\}\}$ and $\theta_{2}$ satisfies $\{s, v\} \vee\{s, w, x\}, \theta_{1} \theta_{2} \theta_{3}$ satisfies $\varphi_{1}$. Similarly, we argue that every $\varphi$-satisfying tactic of an arbitrary $\underset{\otimes}{2}$-induced trace $w$ is of the form $\theta_{1} \theta_{2} \theta_{3}$, and we can show that $w$ is then also of the form $w_{1} w_{2} w_{3}$ as above, yielding satisfaction of $\varphi_{1}$. In contrast to that, $\varphi_{2}$ and $\varphi_{3}$ are

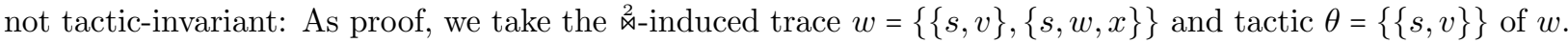
Then, $w \neq \varphi_{2}$ but $\theta \vDash \varphi_{2}$, and $w \vDash \varphi_{3}$ and $\theta \neq \varphi_{3}$.

Given an alignment $\bowtie: \Lambda_{1} \otimes \Lambda_{2}$ that relates the $\kappa_{1}$-groups of $\Lambda_{1}$ with the $\kappa_{2}$-groups of $\Lambda_{2}$, every formula $\varphi_{1} \in \operatorname{LTL}\left[\kappa_{1}\right]$ is aligned to a similar formula $\varphi_{2} \in \operatorname{LTL}\left[\kappa_{2}\right]$. Formulae $\varphi_{1}$ and $\varphi_{2}$ are aligned if $\varphi_{1}$ and $\varphi_{2}$ have the same structure, and $\varphi_{2}$ replaces each disjunction of atomic propositions from $\varphi_{1}$ with an aligned disjunction of atomic propositions. Intuitively, two aligned formulae define the same property (modulo the alignment).

Definition 3.9 (Alignment of LTL-Formulae). Let $\bowtie$ be an alignment. LTL-Formulae $\varphi_{1} \in \operatorname{LTL}[\dot{\ltimes}]$ and $\varphi_{2} \in \mathrm{LTL}\left[\begin{array}{l}\circledR \\ \hdashline\end{array}\right]$ are aligned by $\bowtie$, denoted by $\varphi_{1} \bowtie \varphi_{2}$, iff at least one of the following holds:

- For $i \in\{1,2\}, \varphi_{i}=\bigvee_{K_{i} \in \mathcal{K}_{i}} K_{i}$, where $\mathcal{K}_{i} \subseteq \dot{凶}$ and $\left(\mathcal{K}_{1} \times \grave{凶}\right) \cap \bowtie=\left(\dot{\bowtie} \times \mathcal{K}_{2}\right) \cap \bowtie$.

- For $i \in\{1,2\}, \varphi_{i}=\neg \psi_{i}$ and $\psi_{1} \bowtie \psi_{2}$.

- For $i \in\{1,2\}, \varphi_{i}=\psi_{i} * \psi_{i}^{\prime}$, where $* \in\{\vee, \mathrm{U}\}, \psi_{1} \bowtie \psi_{2}$, and $\psi_{1}^{\prime} \bowtie \psi_{2}^{\prime}$.

For example, given formulae $\varphi_{1}:=(\{a\} \vee\{b, c\}) \cup\{d, e\}$ and $\varphi_{2}:=(\{s, v\} \vee\{t, u\}) \cup\{s, w, x\}$, it holds that $\varphi_{1} \bowtie \varphi_{2}$, where $\bowtie$ is the alignment from Figure 1

\section{Main Results}

This section summarizes the main results of this paper. For each proposed formal statement, we refer to the Lemmata that proves the statement.

First, we turn to one of the fundamental questions regarding an isotactics notion: is it a proper generalisation of the well-established behavioural equivalences? That is, once an alignment collapses to a bijection between the labels of two sets of runs, isotactics shall be grounded in a well-established notion of behavioural equivalence. This is indeed the case, as the following theorem demonstrates a relation between isotactics and trace equivalence - a widely established notion of behavioural equivalence - for repetition-free sets of runs.

Theorem 4.1 (Trace equivalence and isotactics for simple alignments and repetition-free runs). For $i \in\{1,2\}$, let $\Lambda_{i}$ be a set of labels and let $\Sigma_{i} \subseteq \Lambda_{i}^{*}$ be repetition-free. Let $b$ be a bijection from $\Lambda_{1}$ to $\Lambda_{2}$, and let $\bowtie:=\left\{(\{\lambda\},\{b(\lambda)\}) \mid \lambda \in \Lambda_{1}\right\}$. Then, the following statements are equivalent:

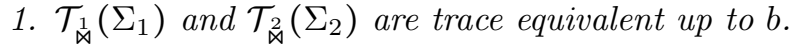

2. $\Sigma_{1} \doteqdot_{\infty} \Sigma_{2}$.

Proof. Follows from Lemmata 5.1 and 5.2 . 
Second, we focus on the question of which system properties are preserved by isotactics. An answer to this question is given for the aforementioned class of tactic-invariant LTL-formulae. Once the choice of a tactic does not affect the truth value of a formula, it is indeed preserved by the proposed notion of equivalence.

Theorem 4.2 (Tactic-invariant LTL-formulae are preserved). Let $\bowtie: \Lambda_{1} \otimes \Lambda_{2}$ be an alignment. For $i \in\{1,2\}$, let $\Sigma_{i} \subseteq \Lambda_{i}^{*}$ and let $\varphi_{i} \in \operatorname{LTL}[\dot{i}]$ be tactic-invariant w.r.t. $\Lambda_{i}$ and $\dot{i}$. Let $\varphi_{1} \bowtie \varphi_{2}$ and let $\Sigma_{1} \doteqdot \Sigma_{2}$. Then, it holds that $\left(\Sigma_{1}, \stackrel{1}{\bowtie}\right) \vDash \varphi_{1}$ iff $\left(\Sigma_{2}, \stackrel{2}{\bowtie}\right) \vDash \varphi_{2}$.

Proof. Follows from Definition 3.7 and Lemma 6.1.

Theorem 4.2 implies that two isotactic (w.r.t. an alignment) FSMs have the same tactic-invariant properties (modulo the alignment). In order to use this result, it must be decidable whether a given LTL-formula is tactic-invariant. The next theorem establishes that this is indeed the case.

Theorem 4.3 (Tactic-invariance of LTL-formula is decidable). Let $\Lambda$ be a set of labels, let $\kappa \subseteq \wp_{>0}(\Lambda)$ be a grouping, and let $\varphi \in \mathrm{LTL}[\kappa]$. Then, the following problem is decidable: To decide whether $\varphi$ is tactic-invariant w.r.t. $\Lambda$ and $\kappa$.

Proof. Follows from Lemmata 6.2 and 6.3 .

Finally, given two FSMs, it is decidable whether their languages are in the interleaving isotactics relation, i.e., it is decidable whether the FSMs are isotactic.

Theorem 4.4 (Isotactics is decidable for FSMs). For $i \in\{1,2\}$, let $\Lambda_{i}$ be a set of labels and let $S_{i}$ be an FSM over $\Lambda_{i}$. Let $\bowtie: \Lambda_{1} \otimes \Lambda_{2}$ be an alignment. Then, the following problem is decidable: To decide whether $\mathcal{L}\left(S_{1}\right)$ and $\mathcal{L}\left(S_{2}\right)$ are in the interleaving isotactics relation w.r.t. $\bowtie$, i.e., to decide whether it holds that $\mathcal{L}\left(S_{1}\right) \doteqdot{ }_{\bowtie} \mathcal{L}\left(S_{2}\right)$.

Proof. Follows from Lemmata 7.8 and 7.9 .

This paper focuses on the definition of isotactics, its preserved properties, and the decidability of the respective verification problem. However, the constructions presented to prove Theorem 4.4 also reveal a decision procedure for isotactics. We implemented this procedure in an open-source tool, which enables a first practical application of isotactics.

\section{Conditional Coincidence of Trace Equivalence and Isotactics}

This section lists two statements that correspond to the two directions of the equivalence stated in Theorem 4.1. These statements justify the proposed conditional coincidence of trace equivalence and isotactics. Let $\bowtie: \Lambda_{1} \otimes \Lambda_{2}$ be an alignment such that $\bowtie$ is a bijection from the singletons over $\Lambda_{1}$ to the singletons over $\Lambda_{2}$. In the proofs, we exploit the fact that every trace $w:=\mathcal{T}_{\dot{⿱}}(\sigma)$, where $i \in\{1,2\}$ and $\sigma \in \Lambda_{i}^{*}$, is a sequence of singletons and, thus, has exactly one tactic, namely $w$.

First, we show that trace equivalence implies isotactics.

Lemma 5.1. For $i \in\{1,2\}$, let $\Lambda_{i}$ be a set of labels and let $\Sigma_{i} \subseteq \Lambda_{i}^{*}$. Let $b$ be a bijection from $\Lambda_{1}$ to $\Lambda_{2}$, and let $\bowtie:=\left\{(\{\lambda\},\{b(\lambda)\}) \mid \lambda \in \Lambda_{1}\right\}$. If $\mathcal{T}_{\bowtie}\left(\Sigma_{1}\right)$ and $\mathcal{T}_{\bowtie}\left(\Sigma_{2}\right)$ are trace equivalent up to $b$, then $\Sigma_{1} \doteqdot_{\bowtie} \Sigma_{2}$

Second, we demonstrate the converse of Lemma 5.1 for the case when $\Sigma_{1}$ and $\Sigma_{2}$ are repetition-free. If $\Sigma_{1}$ and $\Sigma_{2}$ are repetition-free, then the traces induced by the runs in $\Sigma_{1}$ and $\Sigma_{2}$ are repetition-free as well. Consequently, every equivalence class of an equivalence relation induced by a tactic of any of the induced traces is a singleton. Our proof of the next lemma draws on this observation.

Lemma 5.2. For $i \in\{1,2\}$, let $\Lambda_{i}$ be a set of labels and let $\Sigma_{i} \subseteq \Lambda_{i}^{*}$ be repetition-free. Let $b$ be a bijection from $\Lambda_{1}$ to $\Lambda_{2}$, and let $\bowtie:=\left\{(\{\lambda\},\{b(\lambda)\}) \mid \lambda \in \Lambda_{1}\right\}$. If $\Sigma_{1} \doteqdot_{\bowtie} \Sigma_{2}$, then $\mathcal{T}_{\bowtie}\left(\Sigma_{1}\right)$ and $\mathcal{T}_{凶}\left(\Sigma_{2}\right)$ are trace equivalent up to b. ,

The proofs of Lemmata 5.1 and 5.2 are in Appendix A. 


\section{Property Preservation}

Two isotactic collections of traces enjoy the same tactic-invariant LTL properties, cf. Theorem 4.2, while the problem of checking whether a given LTL-formula is tactic-invariant is decidable, cf. Theorem 4.3 These results are due to the lemmata proposed in this section; the proofs of all the statements proposed below are in Appendix A. Next, we argue that for any two aligned traces $w_{1}$ and $w_{2}$, it holds that $w_{1} \vDash \varphi_{1}$ iff $w_{2} \vDash \varphi_{2}$, where $\varphi_{1}$ and $\varphi_{2}$ are aligned tactic-invariant LTL-formulae.

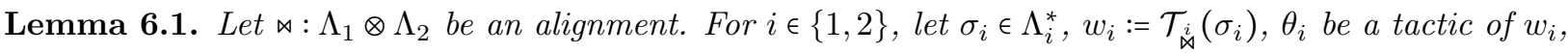
and let $\varphi_{i} \in \mathrm{LTL}[\dot{\dot{\varpi}}]$ be tactic-invariant w.r.t. $\Lambda_{i}$ and $\dot{\aleph}$. Let $\varphi_{1}$ and $\varphi_{2}$ be aligned by $\bowtie$, i.e., $\varphi_{1} \bowtie \varphi_{2}$, and let $\left(\theta_{1}, \theta_{2}\right): w_{1} \bowtie w_{2}$. Then, it holds that $w_{1} \vDash \varphi_{1}$ iff $w_{2} \vDash \varphi_{2}$.

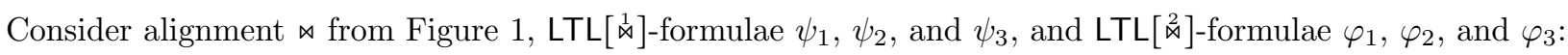

$$
\begin{array}{lll}
\psi_{1}:=\{b, c\} \cup(\{a\} \vee\{d, e\}), & \psi_{2}:=\neg\{a\} \vee \neg\{d, e\}, & \psi_{3}:=\neg \psi_{2}, \\
\varphi_{1}:=\{t, u\} \cup(\{s, v\} \vee\{s, w, x\}), & \varphi_{2}:=\neg\{s, v\} \vee \neg\{s, w, x\}, & \varphi_{3}:=\neg \varphi_{2} .
\end{array}
$$

For all $i \in\{1,2,3\}, \psi_{i}$ and $\varphi_{i}$ are aligned by $\bowtie$, i.e., it holds that $\psi_{i} \bowtie \varphi_{i}$. It is easy to see that $\psi_{1}, \psi_{2}$, and $\psi_{3}$ are tactic-invariant; note that groups in $\dot{\star}$ are pairwise disjoint. Recall from Section 3.4 that $\varphi_{1}$ is tactic-invariant. Therefore, $\psi_{1}$ and $\varphi_{1}$ are 'preserved' for the aligned traces. Let $w_{1}$ be a $\bowtie$-induced trace of the form $\{\{b, c\}\}^{*}(\{\{a\}\} \mid\{\{d, e\}\})$. Then, it holds that $w_{1} \vDash \psi_{1}$. Let $w_{2}$ be a $\ddot{\star}$-induced trace, such that $w_{1}$ and $w_{2}$ can be aligned w.r.t. $\bowtie$. Then, $w_{2}$ is of the form $\{\{t, u\}\}^{*}(\{\{s, v\}\}|\{\{s, w, x\}\}|\{\{s, v\},\{s, w, x\}\})^{+}$, and, thus, it holds that $w_{2} \vDash \varphi_{1}$. Also, recall from Section 3.4 that $\varphi_{2}$ and $\varphi_{3}$ are not tactic-invariant. Next,

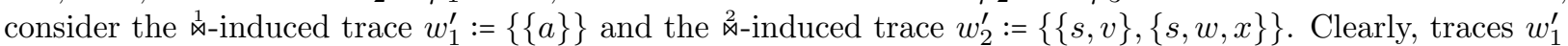
and $w_{2}^{\prime}$ can be aligned w.r.t. $\bowtie$. However, it holds that $w_{1}^{\prime} \vDash \psi_{2}, w_{2}^{\prime} \vDash \varphi_{2}, w_{1}^{\prime} \neq \psi_{3}$, and $w_{2}^{\prime} \vDash \varphi_{3}$.

We reduce the problem of deciding tactic-invariance of a given LTL-formula to the problem of checking language equivalence. To this end, we exploit the fact that every LTL-formula $\varphi \in \operatorname{LTL}[\kappa]$, where $\kappa \subseteq \wp_{>0}(\Lambda)$ is a grouping, can be translated into a corresponding $\operatorname{FSM~}_{\varphi}$. Then, one should intersect the language of $S_{\varphi}$ with the sets of traces and tactics over $\kappa$, respectively, and compare the results.

For the remainder of this section, we fix a grouping $\kappa$ and an LTL-formula $\varphi \in \operatorname{LTL}[\kappa]$.

For some $\kappa$-trace $w$, we write $\operatorname{Tactics}(w)$ to denote the set of all tactics of $w$. Moreover, we introduce the helper sets $W:=\left\{w \in \mathcal{T}_{\kappa}\left(\Lambda^{*}\right) \mid w \vDash \varphi\right\}$ and $\Theta:=\{\theta \in \operatorname{Tactics}(w) \mid w \in W, \theta \vDash \varphi\}$, denoting the sets of all $\varphi$-satisfying $\kappa$-induced traces, and $\varphi$-satisfying tactics of $\kappa$-induced traces in $W$, respectively. A tactic does not carry sufficient information about its 'origin'; there can exist two $\kappa$-induced traces $w, w^{\prime}$ with common tactics, i.e., $\operatorname{Tactics}(w) \cap \operatorname{Tactics}\left(w^{\prime}\right) \neq \varnothing$. For instance, the $\underset{\otimes}{ }$-induced traces $w:=\{\{t, u\}\}\{\{s, v\},\{s, w, x\}\}$ and $w^{\prime}:=\{\{t, u\}\}\{\{s, v\}\}$ have the common tactic $\theta=w^{\prime}$. Therefore, for each tactic $\theta \in \operatorname{Tactics}(w)$ of a $\kappa$-induced trace $w$, we define the sequence $\theta_{w} \in\left(\wp_{=1}(\kappa) \times \wp_{>0}(\kappa)\right)^{*}$ of pairs of sets of groups by $\theta_{w}(i):=(\theta(i), w(i))$, $i \in\{1, \ldots,|w|\}$. That is, each element of $\theta_{w}$ at position $i$ refers to the group chosen by tactic $\theta$ and the set of groups this group has been chosen from. For instance, for the $\underset{\otimes}{2}$-induced trace $w:=\{\{t, u\}\}\{\{s, v\},\{s, w, x\}\}$ and its tactic $\theta:=\{\{t, u\}\}\{\{s, v\}\}$, it holds that $\theta_{w}=(\{\{t, u\}\},\{\{t, u\}\})(\{\{s, v\}\},\{\{s, v\},\{s, w, x\}\})$. We refer to $\theta_{w}$ as the enriched version of tactic $\theta$.

Based on the notion of $\theta_{w}$, we define the sets $\hat{\Theta}:=\left\{\theta_{w} \mid w \in W, \theta \in \operatorname{Tactics}(w)\right\}$ and $\hat{\Theta}^{\prime}:=\left\{\theta_{w} \mid \theta \in \Theta, w \epsilon\right.$ $\left.\mathcal{T}_{\kappa}\left(\Lambda^{*}\right)\right\}$ built from $W$ and $\Theta$, respectively. That is, $\hat{\Theta}$ contains the enriched versions of all the tactics of $\varphi$-satisfying traces, and $\hat{\Theta}^{\prime}$ contains the enriched versions of all the $\varphi$-satisfying tactics of arbitrary traces.

Next, we demonstrate that tactic-invariance coincides with equality of $\hat{\Theta}$ and $\hat{\Theta}^{\prime}:$ If $\varphi$ is tactic-invariant, then for every trace $w$ and tactic $\theta \in \operatorname{Tactics}(w)$, it holds that $w \in W$ iff $\theta \in \Theta$. Otherwise, there exist a trace $w$ and a tactic $\theta \in \operatorname{Tactics}(w)$ such that $w \in W$ iff $\theta \notin \Theta$.

Lemma 6.2. $\hat{\Theta}=\hat{\Theta}^{\prime}$ iff $\varphi$ is tactic-invariant w.r.t. $\Lambda$ and $\kappa$.

For example, consider the 2 -induced trace $w:=\{\{s, v\},\{s, w, x\}\}$ and tactic $\theta:=\{\{s, v\}\}$ of $w$, yielding $\theta_{w}=(\{\{s, v\}\},\{\{s, v\},\{s, w, x\}\})$. As mentioned above, $w \neq \varphi_{2}$ and $w \vDash \varphi_{3}$; note that $w=w_{2}^{\prime}$. But, it holds that $\theta \vDash \varphi_{2}$ and $\theta \notin \varphi_{3}$. Assuming that $\varphi=\varphi_{2}$, it holds that $\theta_{w} \notin \hat{\Theta}$; note that $w \notin W$. However, it holds that 


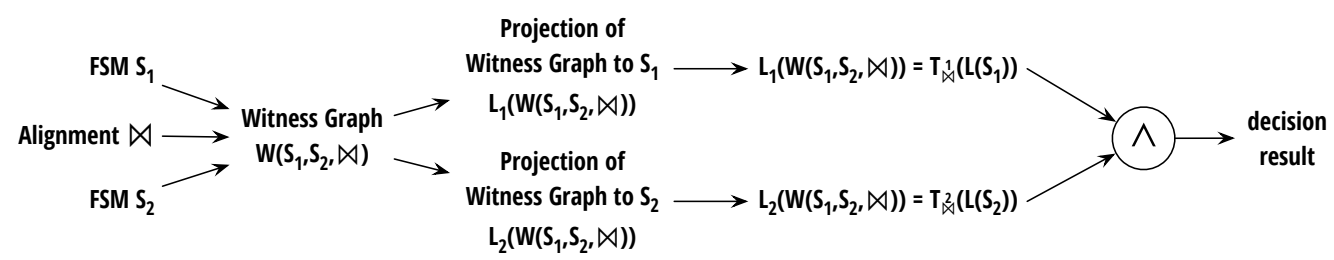

Figure 4. A schematic view of the proposed approach for deciding interleaving isotactics.

$\theta_{w} \in \hat{\Theta}^{\prime}$, because $\theta \vDash \varphi_{2}$. Similarly, assuming that $\varphi=\varphi_{3}$, one can check that $\theta_{w} \in \hat{\Theta}$ and $\theta_{w} \notin \hat{\Theta}^{\prime}$. According to Lemma 6.2. these facts confirm that $\varphi_{2}$ and $\varphi_{3}$ are not tactic-invariant.

We reduce the decision of whether $\hat{\Theta}=\hat{\Theta}^{\prime}$ to the check of language equivalence of FSMs, thus demonstrating decidability of the equality and, consequently, of tactic-invariance. The idea is to construct FSMs $\hat{S}_{\Theta}$ and $\hat{S}_{\Theta}^{\prime}$ that accept $\hat{\Theta}$ and $\hat{\Theta}^{\prime}$, respectively. To this end, we construct FSMs $S_{W}$ and $S_{\Theta}$ that accept $W$ and $\Theta$, respectively; here, we exploit the fact that $\varphi$ can be encoded as FSM $S_{\varphi}$. Then, we 'unfold' the transitions of $S_{W}$ and $S_{\Theta}$ to obtain $\hat{S}_{\Theta}$ and $\hat{S}_{\Theta}^{\prime}$, respectively. Finally, we decide $\hat{\Theta}=\hat{\Theta}^{\prime}$ by deciding language equivalence of $\hat{S}_{\Theta}$ and $\hat{S}_{\Theta}^{\prime}$.

Lemma 6.3. It is decidable whether $\hat{\Theta}$ and $\hat{\Theta}^{\prime}$ are equal sets.

Assuming that $\varphi=\varphi_{1}$, Appendix B exemplifies the construction of $S_{\varphi}, S_{W}, S_{\Theta}, \hat{S}_{\Theta}$, and $\hat{S}_{\Theta}^{\prime}$.

Deciding tactic-invariance of $\varphi$ requires at most exponential space w.r.t. to the size of $\varphi$ and $\bowtie$ : The FSM $S_{\varphi}$ can be computed in EXPTIME and has exponential size w.r.t. $\varphi$ and $\bowtie$. The FSMs $\hat{S}_{\Theta}$ and $\hat{S}_{\Theta}^{\prime}$ can be computed from $S_{\varphi}$ in polynomial time; the resulting FSMs having exponential size. Finally, the equivalence check requires polynomial space w.r.t. the size of the exponentially-sized FSMs.

\section{Deciding Isotactics}

In this section, we demonstrate the decidability of interleaving isotactics for languages of FSMs $S_{1}$ and $S_{2}$ w.r.t. an alignment $\bowtie$. The idea of the proposed approach is shown in Figure 4 . It comprises three steps:

1. Compute the 'product' $\mathcal{W}\left(S_{1}, S_{2}, \bowtie\right)$ of $S_{1}$ and $S_{2}$ w.r.t. $\bowtie$, called witness graph.

2. For $i \in\{1,2\}$, compute the 'projection' $\mathcal{L}_{i}\left(\mathcal{W}\left(S_{1}, S_{2}, \bowtie\right)\right)$ of $\mathcal{W}\left(S_{1}, S_{2}, \bowtie\right)$, describing the behaviour of $S_{i}$ that can be mirrored by $S_{j}, j \in\{1,2\}, j \neq i$.

3. Reduce deciding interleaving isotactics to a language equivalence check, that is, the equality of the

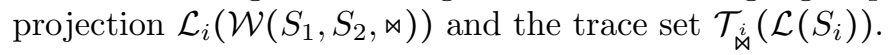

Again, the proofs of the main formal statements proposed in this section are in Appendix A

Determinism. To simplify subsequent discussions, we assume that $S_{1}$ and $S_{2}$ are deterministic w.r.t. $\grave{\aleph}$ and $\underset{\star}{2}$, respectively. Here, determinism is defined w.r.t. a given grouping, and requires the absence of labels which do not participate in any group.

Definition 7.1 (Deterministic FSM). An FSM $S:=\left(\mathcal{Q}, \Lambda, \rightarrow, q^{i n i}, F\right)$ is deterministic w.r.t. a grouping $\kappa$ of $\Lambda$, iff for all states $q \in \mathcal{Q}$ and for all transitions $q \stackrel{\lambda_{1}}{\longrightarrow} q_{1}$ and $q \stackrel{\lambda_{2}}{\longrightarrow} q_{2}$ of $S$ : If both $\mathcal{G}_{\kappa}\left(\lambda_{1}\right) \neq \varnothing$ and $\mathcal{G}_{\kappa}\left(\lambda_{1}\right)=\mathcal{G}_{\kappa}\left(\lambda_{2}\right)$, then $q_{1}=q_{2}$.

The FSMs $m_{1}$ and $m_{2}$ in Figure 1 are deterministic w.r.t. to $\dot{\star}$ and $\ddot{\star}$, respectively. A counter example for determinism would be if in $m_{1}$ one adds a fresh state 4 and a fresh transition $1 \stackrel{c}{\rightarrow} 4$. The FSM obtained in

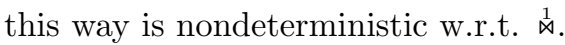

Given an FSM $S$ and a grouping, one can always construct a deterministic FSM w.r.t. the grouping that describes the same set of induced traces as $S$. The construction can be accomplished using the powerset construction [21]. 
Lemma 7.2. Let $S:=\left(\mathcal{Q}, \Lambda, \rightarrow, q^{i n i}, F\right)$ be an FSM, and let $\kappa$ be a grouping of $\Lambda$. There exists an FSM $S^{\prime}$ such that: (i) $\mathcal{T}_{\kappa}(\mathcal{L}(S))=\mathcal{T}_{\kappa}\left(\mathcal{L}\left(S^{\prime}\right)\right)$, and (ii) $S^{\prime}$ is deterministic w.r.t. $\kappa$.

For the remainder of this section, we fix an alignment $\bowtie: \Lambda_{1} \otimes \Lambda_{2}$, and two FSMs $S_{i}:=\left(\mathcal{Q}_{i}, \Lambda_{i}, \rightarrow_{i}, q_{i}^{i n i}, F_{i}\right)$, $i \in\{1,2\}$, such that $S_{i}$ is deterministic w.r.t. $\dot{\star}$.

Matches. The idea behind the witness graph $\mathcal{W}\left(S_{1}, S_{2}, \bowtie\right)$ is to construct a finite representation of all possible ways to align the traces of $S_{1}$ with the traces of $S_{2}$. To this end, we build a product of $S_{1}$ and $S_{2}$ where each product state $\left(q_{1}, q_{2}\right)$ is additionally distinguished by a set $M$ of possible matches. Here, a match is a pair $\left(K_{1}, K_{2}\right)$ of two aligned groups $K_{1}$ and $K_{2}$, indicating that there exist traces $w_{1}$ and $w_{2}$ yielding $q_{1}$ and $q_{2}$, respectively, which can be aligned by some tactics $\theta_{1}$ and $\theta_{2}$ satisfying $\theta_{i}\left(\left|\theta_{i}\right|\right)=\left\{K_{i}\right\}$, for $i \in\{1,2\}$. The edges of $\mathcal{W}\left(S_{1}, S_{2}, \bowtie\right)$ are labelled with pairs $\left(\mathcal{K}_{1}, \mathcal{K}_{2}\right)$, where each $\mathcal{K}_{i}, i \in\{1,2\}$, is a set of groups. A non-empty set of groups $\mathcal{K}_{i}$ indicates an action of $S_{i}$ which is abstracted by $\dot{\star}$ to $\mathcal{K}_{i}$. In contrast to that, $\mathcal{K}_{i}=\varnothing$ indicates that $S_{i}$ did not 'move'.

To simplify further discussions, we introduce the notation $M+\left(\mathcal{K}_{1}, \mathcal{K}_{2}\right)$ for a set $M$ of matches and a pair $\left(\mathcal{K}_{1}, \mathcal{K}_{2}\right)$ of sets of groups. Intuitively, $M+\left(\mathcal{K}_{1}, \mathcal{K}_{2}\right)$ describes the set of possible result matches if we start from $M$, and both FSMs act according to $\left(\mathcal{K}_{1}, \mathcal{K}_{2}\right)$.

Let $M \subseteq \bowtie$ and let $\mathcal{K}_{i} \subseteq \dot{凶}, i \in\{1,2\}$, such that $\mathcal{K}_{1} \cup \mathcal{K}_{2} \neq \varnothing$. Then, $M+\left(\mathcal{K}_{1}, \mathcal{K}_{2}\right)$ is defined as follows:

$$
M+\left(\mathcal{K}_{1}, \mathcal{K}_{2}\right):= \begin{cases}\left(\mathcal{K}_{1} \times \mathcal{K}_{2}\right) \cap \bowtie & \text { if } M=\varnothing \\ \left\{\left(G_{1}, G_{2}\right) \in\left(\left(\mathcal{K}_{1} \times \mathcal{K}_{2}\right) \cap \bowtie\right) \backslash M \mid \exists\left(G_{1}^{\prime}, G_{2}^{\prime}\right) \in M: G_{1} \neq G_{1}^{\prime} \wedge G_{2} \neq G_{2}^{\prime}\right\} & \text { if } \mathcal{K}_{1} \neq \varnothing \wedge \mathcal{K}_{2} \neq \varnothing, \\ \left\{\left(G_{1}, G_{2}\right) \in M \mid \exists i \in\{1,2\}: G_{i} \in \mathcal{K}_{i}\right\} & \text { otherwise. }\end{cases}
$$

Consider $m_{1}, m_{2}$, and $\bowtie$ as defined in Figure 1, and let $M:=\{(\{b, c\},\{t, u\}),(\{d, e\},\{s, w, x\})\}$. Then, it holds that $M+(\{\{a\},\{b, c\}\},\{\{s, v\},\{s, w, x\}\})=\{(\{a\},\{s, v\})\}, M+(\{\{b, c\}\}, \varnothing)=\{(\{b, c\},\{t, u\})\}$, and $M+(\{\{b, c\}\},\{\{t, u\}\})=\varnothing$.

The Witness Graph. Next, we define the witness graph $\mathcal{W}\left(S_{1}, S_{2}, \bowtie\right)$ based on the possible transitions in $S_{1}$ and $S_{2}$. We start from the initial state, and the empty set of matches. Then, we add nodes and edges according to the respective transition relations and the alignment.

Definition 7.3 (Witness Graph). The witness graph $\mathcal{W}\left(S_{1}, S_{2}, \bowtie\right)$ of $S_{1}$ and $S_{2}$ w.r.t. an alignment $\bowtie$ is the least edge-labelled graph $(V, E)$, where $V \subseteq \mathcal{Q}_{1} \times \mathcal{Q}_{2} \times \wp(\bowtie)$ and $E \subseteq V \times \wp($ 冈 $) \times \wp($ ฝ $) \times V$, such that:

1. $\left(q_{1}^{\text {ini }}, q_{2}^{\text {ini }}, \varnothing\right) \in V$.

2. Let $v=\left(q_{1}, q_{2}, M\right) \in V$.

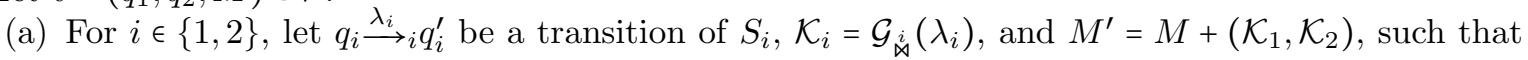
$M^{\prime} \neq \varnothing$. Then, it holds that $v^{\prime}:=\left(q_{1}^{\prime}, q_{2}^{\prime}, M^{\prime}\right) \in V$ and $\left(v, \mathcal{K}_{1}, \mathcal{K}_{2}, v^{\prime}\right) \in E$.

(b) Let $i, j \in\{1,2\}, i \neq j$. Let $q_{i} \stackrel{\lambda_{i}}{\rightarrow} i q_{i}^{\prime}$ be a transition of $S_{i}, \mathcal{K}_{i}=\mathcal{G}_{\dot{\dot{\omega}}}\left(\lambda_{i}\right), \mathcal{K}_{j}=\varnothing, q_{j}^{\prime}=q_{j}$, and $M^{\prime}=$ $M+\left(\mathcal{K}_{1}, \mathcal{K}_{2}\right)$, such that $M^{\prime} \neq \varnothing$. Then, it holds that $v^{\prime}:=\left(q_{1}^{\prime}, q_{2}^{\prime}, M^{\prime}\right) \in V$ and $\left(v, \mathcal{K}_{1}, \mathcal{K}_{2}, v^{\prime}\right) \in E$.

Let $e=\left(v, \mathcal{K}_{1}, \mathcal{K}_{2}, v^{\prime}\right) \in E$. For $i \in\{1,2\}, e[i]:=\mathcal{K}_{i}$ and $e[i]_{\neq \varnothing}:= \begin{cases}e[i] & e[i] \neq \varnothing, \\ \varepsilon & \text { otherwise. }\end{cases}$

Let $v_{0} \ldots v_{n} \in V^{*}$ such that $v_{0}=\left(q_{1}^{i n i}, q_{2}^{i n i}, \varnothing\right)$. Let $\pi=e_{1} \ldots e_{n} \in E^{*}$ such that $\pi(i)=\left(v_{i-1}, \mathcal{K}_{1}^{i}, \mathcal{K}_{2}^{i}, v_{i}\right)$ for all $i \in\{1, \ldots, n\}$. Then, $\pi$ is a path of $\mathcal{W}\left(S_{1}, S_{2}, \bowtie\right)$ resulting in $v_{n}$. We define $\pi[i]:=\pi(1)[i] \ldots \pi(n)[i]$, and $\pi[i]_{\neq \varnothing}:=\pi(1)[i]_{\neq \varnothing} \ldots \pi(n)[i]_{\neq \varnothing}$, where $i \in\{1,2\}$.

Figure 5 exemplifies the notion of the witness graph $\mathcal{W}\left(m_{1}, m_{2}, \bowtie\right)$ for the FSMs $m_{1}$ and $m_{2}$, and the alignment $\bowtie$ given in Figure 1. In the proposed notation, the outmost round brackets in the node labels are omitted. For example, the topmost node in the figure is $(1, \mathrm{I}, \varnothing)$, but we write $1, \mathrm{I}, \varnothing$. Note that edge labels are written in two lines (the first element is written above the second). We also omit the outmost braces when depicting each of the non-empty elements of the label. For example, the edge from $(1, \mathrm{I}, \varnothing)$ to $(1, \mathrm{II},\{(\{a\},\{s, v\})\})$ has label $(\{\{a\}\},\{\{s, v\},\{s, w, x\}\})$. However, in the figure, the corresponding edge 


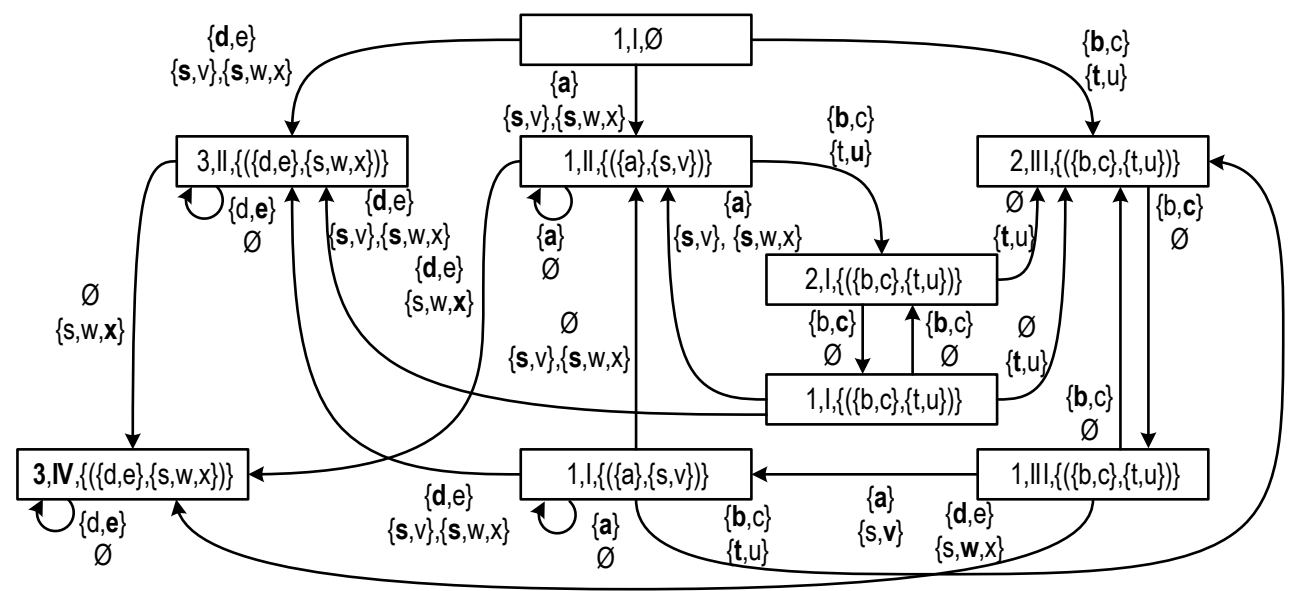

Figure 5. Witness graph for the FSMs and the alignment shown in Figure 1

is labelled with $\{a\}$ written above $\{s, v\},\{s, w, x\}$. Labels of the respective transitions in $m_{1}$ and $m_{2}$ are printed in bold in the edge labels of the witness graph. Finally, if both $m_{1}$ and $m_{2}$ are in a final state, the state names are also put in bold in the graph.

For example, Rule 2(a) in Definition 7.3 produces the edge labelled $(\{\{a\}\},\{\{s, v\},\{s, w, x\}\})$ from node $(1, \mathrm{I}, \varnothing)$ to node $\left(1, \mathrm{II},\{(\{a\},\{s, v\})\}: \stackrel{a^{a}}{\rightarrow} m_{1} 1, \stackrel{s}{\rightarrow} m_{2} \mathrm{II}, \mathcal{G}_{\grave{\downarrow}}(a)=\{\{a\}\}, \mathcal{G}_{\grave{凶}}(s)=\{\{s, v\},\{s, w, x\}\}\right.$,

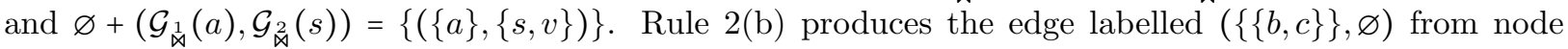
$(2, \mathrm{III},\{(\{b, c\},\{t, u\})\})$ to $(1, \mathrm{III},\{(\{b, c\},\{t, u\})\}): 2 \stackrel{c}{\rightarrow} m_{1} 1, \mathcal{G}_{\text {ฌ }}(c)=\{\{b, c\}\}$, and $\{(\{b, c\},\{t, u\})\}+\left(\mathcal{G}_{\text {ฝ }}(c)\right.$, $\varnothing)=\{(\{b, c\},\{t, u\})\}$. The sequence $\pi$ of edges with respective labels $(\{\{a\}\},\{\{s, v\},\{s, w, x\}\}),(\{\{b, c\}\}$, $\{\{t, u\}\}),(\{\{b, c\}\}, \varnothing)$ and $(\varnothing,\{\{t, u\}\})$ from node $(1, \mathrm{I}, \varnothing)$ to node $(2$ IIII, $\{(\{b, c\},\{t, u\})\})$ is a path with $\pi[2]=\{\{s, v\},\{s, w, x\}\}\{\{t, u\}\} \varnothing\{\{t, u\}\}$ and $\pi[2]_{\neq \varnothing}=\{\{s, v\},\{s, w, x\}\}\{\{t, u\}\}\{\{t, u\}\}$.

For illustration, we sketch an example producing non-singleton sets of matches: For $i \in\{1,2\}$, let $K_{i}$ and $K_{i}^{\prime}$ be groups with $\lambda_{i} \in K_{i} \cap K_{i}^{\prime}$. Let $\bowtie^{\prime}$ be an alignment with $K_{1} \bowtie^{\prime} K_{2}$ and $K_{1}^{\prime} \bowtie K_{2}^{\prime}$. For $i \in\{1,2\}$, let $S_{i}$ be an FSM accepting the word $\lambda_{i}$. Then, $\mathcal{W}\left(S_{1}, S_{2}, \bowtie^{\prime}\right)$ has an edge labelled $\left(\left\{K_{1}, K_{1}^{\prime}\right\},\left\{K_{2}, K_{2}^{\prime}\right\}\right)$ from the initial node to a node with matches $\left\{\left(K_{1}, K_{2}\right),\left(K_{1}^{\prime}, K_{2}^{\prime}\right)\right\}$.

Realisability. A pair $\left(w_{1}, w_{2}\right) \in \wp(\hat{\aleph})^{*} \times \wp(\underset{\aleph}{2})^{*}$ is realisable iff there exists a path of $\mathcal{W}\left(S_{1}, S_{2}, \bowtie\right)$ that represents $w_{1}$ and $w_{2}$, possibly also containing $\varnothing$.

Definition 7.4 (Realisable). For $i \in\{1,2\}$, let $w_{i} \in \wp(\dot{\dot{x}})^{*}$. Then, $\left(w_{1}, w_{2}\right)$ is realisable in $\mathcal{W}\left(S_{1}, S_{2}, \bowtie\right)$ resulting in a node $v$ iff there is a path $\pi$ of $\mathcal{W}\left(S_{1}, S_{2}, \bowtie\right)$ resulting in $v$, such that $\pi[i]_{\neq \varnothing}=w_{i}, i \in\{1,2\}$.

For example, let $w_{1}:=\mathcal{T}_{\text {ฟ }}(b c a)=\{\{b, c\}\}\{\{b, c\}\}\{\{a\}\}$ and $w_{2}:=\mathcal{T}_{\grave{\unrhd}}(t v)=\{\{t, u\}\}\{\{s, v\}\}$. Then, $\left(w_{1}, w_{2}\right)$ is realisable in the graph in Figure 5. resulting in node $(1, \mathrm{I},\{(\{a\},\{s, v\})\})$. In contrast, the pair of traces $\left(\mathcal{T}_{\text {ฌ }}(b c), w_{2}\right)$ is not realisable.

We now show that realisability of $\left(w_{1}, w_{2}\right)$ implies that $w_{1}$ and $w_{2}$ can be aligned.

Lemma 7.5. For $i \in\{1,2\}$, let $w_{i} \in \wp(\dot{\star})^{*}$. Let $\left(w_{1}, w_{2}\right)$ be realisable in $\mathcal{W}\left(S_{1}, S_{2}, \bowtie\right)$ resulting in a node $v=\left(q_{1}, q_{2}, M\right)$. For each $\left(K_{1}, K_{2}\right) \in M$, there exist a tactic $\theta_{1}$ of $w_{1}$ and a tactic $\theta_{2}$ of $w_{2}$, such that $\left(\theta_{1}, \theta_{2}\right): w_{1} \bowtie w_{2}$ and for all $i \in\{1,2\}:\left|w_{i}\right|>0 \Rightarrow \theta_{i}\left(\left|w_{i}\right|\right)=\left\{K_{i}\right\}$.

Because $\left(w_{1}, w_{2}\right)$ is realizable, where $w_{1}:=\mathcal{T}_{\grave{\aleph}}(b c a)=\{\{b, c\}\}\{\{b, c\}\}\{\{a\}\}$ and $w_{2}:=\mathcal{T}_{\grave{凶}}(t v)=\{\{t, u\}\}\{\{s, v\}\}$, $w_{1}$ and $w_{2}$ can be aligned. To justify this fact, one can use tactics $\theta_{1}:=w_{1}$ and $\theta_{2}:=w_{2}$, and the bijection $\{\{1,2\} \mapsto\{1\},\{3\} \mapsto\{2\}\}$ from $\left\{1, \ldots,\left|\theta_{1}\right|\right\} / \theta_{1}$ to $\left\{1, \ldots,\left|\theta_{2}\right|\right\} / \theta_{2}$.

We now show the converse, i.e., if $w_{1}$ and $w_{2}$ can be aligned, then $\left(w_{1}, w_{2}\right)$ is realisable. The idea of the proof is to construct a path that justifies that $\left(w_{1}, w_{2}\right)$ is indeed realisable based on alignable tactics $\theta_{1}$ and 
$\theta_{2}$ in the order of the aligned equivalence classes: Rule 2(a) creates an edge for every fresh pair of aligned equivalence classes, while Rule 2(b) creates the edges for the remaining indices in these equivalence classes.

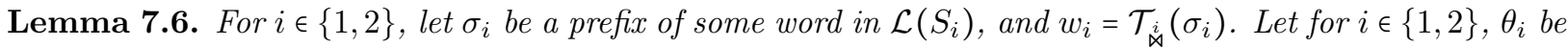
a tactic of $w_{i}$, such that $\left(\theta_{1}, \theta_{2}\right): w_{1} \bowtie w_{2}$. Then, $\left(w_{1}, w_{2}\right)$ is realisable resulting in some node $v=\left(q_{1}, q_{2}, M\right)$ with $\left|w_{1}\right|>0 \Rightarrow\left|w_{2}\right|=0 \wedge\left(\theta_{1}\left(\left|w_{1}\right|\right), \theta_{2}\left(\left|w_{2}\right|\right)\right) \in M$.

For illustration, we again consider $w_{1}:=\mathcal{T}_{\grave{凶}}(b c a)=\{\{b, c\}\}\{\{b, c\}\}\{\{a\}\}$ and $w_{2}:=\mathcal{T}_{\vec{凶}}(t v)=\{\{t, u\}\}\{\{s, v\}\}$, which can be aligned by tactics $\theta_{1}:=w_{1}$ and $\theta_{2}:=w_{2}$, and bijection $\{\{1,2\} \mapsto\{1\},\{3\} \mapsto\{2\}\}$. One can construct a path that justifies that $\left(w_{1}, w_{2}\right)$ is realisable by using the bijection: $\{1,2\} \mapsto\{1\}$ yields that one must start by first taking edge $\left(\theta_{1}(1), \theta_{2}(1)\right)$ followed by edge $\left(\theta_{1}(2), \varnothing\right)$. Then, $\{3\} \mapsto\{2\}$ yields edge $\left(\theta_{1}(3), \theta_{2}(2)\right)$.

Projecting the Witness Graph. Intuitively, for $i, j \in\{1,2\}, i \neq j$, we can conceive $\mathcal{W}\left(S_{1}, S_{2}, \bowtie\right)$ as an FSM, where for each edge labelled $\left(\mathcal{K}_{1}, \mathcal{K}_{2}\right)$, we only consider the action $\mathcal{K}_{i}$ of $S_{i}$, i.e., we omit the actions of $S_{j}$ :

Definition 7.7 (Witness Graph Projection). Let $\Pi$ be the set of all paths of $\mathcal{W}\left(S_{1}, S_{2}, \bowtie\right)$ resulting in a node $\left(q_{1}, q_{2}, M\right)$, where $q_{1} \in F_{1}$ and $q_{2} \in F_{2}$. For $i \in\{1,2\}$, we define $\mathcal{L}_{i}\left(\mathcal{W}\left(S_{1}, S_{2}, \bowtie\right)\right):=\left\{\pi[i]_{\neq \varnothing} \mid \pi \in \Pi\right\}$.

From Lemma 7.6 and Lemma 7.5, we get that $\mathcal{L}_{i}\left(\mathcal{W}\left(S_{1}, S_{2}, \bowtie\right)\right)$ describes the behavior of $S_{i}$ which can be aligned to behavior of $S_{j}$. This enables us to reduce deciding isotactics to comparing $S_{i}$ to $\mathcal{L}_{i}\left(\mathcal{W}\left(S_{1}, S_{2}, \bowtie\right)\right)$.

Reducing Decidability of Isotactics to Language Equivalence. We reduce the problem of deciding isotactics of $\mathcal{L}\left(S_{1}\right)$ and $\mathcal{L}\left(S_{2}\right)$ to two language equivalence checks, between the FSMs and the respective projections of the witness graph.

Lemma 7.8. The following statements are equivalent:

1. $\mathcal{L}_{1}\left(\mathcal{W}\left(S_{1}, S_{2}, \bowtie\right)\right)=\mathcal{T}_{\text {ฌ }}\left(\mathcal{L}\left(S_{1}\right)\right)$ and $\mathcal{L}_{2}\left(\mathcal{W}\left(S_{1}, S_{2}, \bowtie\right)\right)=\mathcal{T}_{\grave{\unrhd}}\left(\mathcal{L}\left(S_{2}\right)\right)$.

2. $\mathcal{L}\left(S_{1}\right) \doteqdot_{\bowtie} \mathcal{L}\left(S_{2}\right)$.

Because language equivalence is decidable for FSMs [21], we can also decide the first proposition of Lemma 7.8 by transforming $\mathcal{W}\left(S_{1}, S_{2}, \bowtie\right)$ into two FSMs: One with language $\mathcal{L}_{1}\left(\mathcal{W}\left(S_{1}, S_{2}, \bowtie\right)\right)$ and the other with language $\mathcal{L}_{2}\left(\mathcal{W}\left(S_{1}, S_{2}, \bowtie\right)\right)$. The transformation basically comprises the projection of the edge labels to the $i$-th component, $i \in\{1,2\}$, and the subsequent "removal" of $\varnothing$-transitions.

Lemma 7.9. For $i \in\{1,2\}$, the following problem is decidable:

To decide whether it holds that $\mathcal{L}_{i}\left(\mathcal{W}\left(S_{1}, S_{2}, \bowtie\right)\right)=\mathcal{T}_{\dot{\dot{亠}}}\left(\mathcal{L}\left(S_{i}\right)\right)$.

We conclude that deciding isotactics is in EXSPACE: If the FSMs are deterministic, $\mathcal{W}\left(S_{1}, S_{2}, \bowtie\right)$ has at most $\left|\mathcal{Q}_{1}\right| \cdot\left|\mathcal{Q}_{2}\right| \cdot 2^{|\bowtie|}$ nodes; otherwise, determinisation of the FSMs yields a witness graph with at most $2^{\left|\mathcal{Q}_{1}\left\|\mathcal{Q}_{2}\right\| \bowtie\right|}$ nodes. $\mathcal{W}\left(S_{1}, S_{2}, \bowtie\right)$ can be computed in EXPTIME. Deciding language equivalence requires polynomial space in the size of the FSMs.

\section{Concluding Remarks}

This paper proposed interleaving isotactics to assess behavioural equivalence of aligned models, presented results on the properties it preserves, and proved decidability of the respective verification problems. The constructions introduced in Section 7 to show decidability of isotactics, i.e., the witness graph and its projections, give rise to a first decision procedure for isotactics. This procedure has been implemented and is available in an open-source tool. $]^{4}$ It takes as input two FSMs and the definition of an alignment and returns a Boolean result. It also enables the creation of visualisations of the constructed witness graph and its projections and comes with exemplary input files that encode the running example of this paper.

${ }^{4}$ https://github.com/Isotactics/deciding-isotactics 
Having introduced a novel equivalence notion, we also reflect on potential causes for non-equivalence. First and foremost, an action that is part solely of one model may lead to non-equivalence. In our model, this situation may manifest when the label of such an action is related to itself in the alignment. As occurrences of this label are limited to runs of one of the system models, they cannot be mirrored by any run of the other model. Assessment of behavioural equivalence of system models often starts by hiding non-matching actions, i.e., by making non-matching actions silent, or invisible. Then, models are compared based on the remaining visible actions, see for example weak trace equivalence 11. Isotactics is a generalization of (notions like) weak trace equivalence. One can implement action hiding using the isotactics' alignment relation, by excluding the labels of non-matching actions from the alignment. Note that weak trace equivalence cannot capture behavioural correspondence of an action in one model with two distinct mutually exclusive actions in the other model. This correspondence, however, can be captured in an alignment relation and verified using isotactics. Finally, the notion of behaviour inheritance suggests that when verifying an equivalence, in addition to being hidden, an action can be blocked [2. That is, the blocked action, and all the subsequent actions, are considered to be not reachable. This idea, originally proposed in the context of branching bisimulation, can be lifted to isotactics in a straightforward manner.

For system models that are not behaviourally equivalent, it is often relevant to quantify the discrepancies in their behaviour. To this end, measures for behavioural similarity have been proposed for the different semantics of system models, see [7, 8. We foresee that the proposed notion of isotactics can be exploited for the definition of similar measures. For instance, such new measures can aim to quantify the ratio of the aligned groups of labels for which the behavioural projections are equivalent.

\section{Acknowledgements}

We are grateful for the support by the Universities Australia (UA) and the German Academic Exchange Service (DAAD) as part of the Joint Research Co-operation Scheme. Artem Polyvyanyy was partly supported by the Australian Research Council Discovery Project DP180102839. We further would like to thank Frank Lange and Sebastian Misch for their work on the implementation of the isotactics decision procedure.

\section{References}

[1] Christel Baier and Joost-Pieter Katoen. Principles of Model Checking. MIT Press, 2008.

[2] Twan Basten and Wil M. P. van der Aalst. Inheritance of behavior. J. Logic. Algebr. Program., 47(2):47-145, 2001. doi:10.1016/S1567-8326(00)00004-7

[3] Eike Best, Raymond R. Devillers, Astrid Kiehn, and Lucia Pomello. Concurrent bisimulations in Petri nets. Acta Inform. 28(3):231-264, 1991. doi:10.1007/BF01178506

[4] Moisés Castelo Branco, Javier Troya, Krzysztof Czarnecki, Jochen Malte Küster, and Hagen Völzer. Matching business process workflows across abstraction levels. In MoDELS, pages 626-641, 2012. doi:10.1007/978-3-642-33666-9_40

[5] Wilfried Brauer, Robert Gold, and Walter Vogler. A survey of behaviour and equivalence preserving refinements of Petri nets. In ATPN, pages 1-46, 1989. doi:10.1007/3-540-53863-1_19

[6] Michael C. Browne, Edmund M. Clarke, and Orna Grumberg. Characterizing finite Kripke structures in propositional temporal logic. Theor. Comput. Sci., 59:115-131, 1988. doi:10.1016/0304-3975(88)90098-9

[7] Luca de Alfaro, Marco Faella, and Mariëlle Stoelinga. Linear and branching system metrics. IEEE Trans. Softw. Eng., 35(2):258-273, 2009. doi:10.1109/TSE.2008.106

[8] Josee Desharnais, Vineet Gupta, Radha Jagadeesan, and Prakash Panangaden. Metrics for labelled Markov processes. Theor. Comput. Sci., 318(3):323-354, 2004. doi:10.1016/j.tcs.2003.09.013

[9] V. M. Glushkov. The abstract theory of automata. Russian Mathematical Surveys, 16(5):1-53, 1961. doi:10.1070/ RM1961v016n05ABEH004112.

[10] Volker Guth and Andreas Oberweis. Delta-analysis of Petri net based models for business processes. In International Conference on Applied Informatics, pages 23-32, 1997.

[11] Petr Jancar, Javier Esparza, and Faron Moller. Petri nets and regular processes. J. Comput. Syst. Sci., 59(3):476-503, 1999. doi:10.1006/jcss.1999.1643

[12] Michael Kaminski. Invariance under stuttering in a temporal logic of actions. Theor. Comput. Sci., 368(1-2):50-63, 2006. doi:10.1016/j.tcs.2006.06.020

[13] Donald E. Knuth. The Art of Computer Programming, Volume I: Fundamental Algorithms. Addison-Wesley, 1968.

[14] Martin Lange, Étienne Lozes, and Manuel Vargas Guzmán. Model-checking process equivalences. Theor. Comput. Sci., 560:326-347, 2014. doi:10.1016/j.tcs.2014.08.020 
[15] Doron A. Peled and Thomas Wilke. Stutter-invariant temporal properties are expressible without the next-time operator. Inf. Process. Lett., 63(5):243-246, 1997. doi:10.1016/S0020-0190(97)00133-6

[16] Amir Pnueli. The temporal logic of programs. In FOCS, pages 46-57, 1977. doi:10.1109/SFCS.1977.32.

[17] Artem Polyvyanyy, Chun Ouyang, Alistair Barros, and Wil M. P. van der Aalst. Process querying: Enabling business intelligence through query-based process analytics. Decis. Support Syst., 100:41-56, 2017. doi:10.1016/j.dss.2017.04.011

[18] Artem Polyvyanyy, Matthias Weidlich, and Mathias Weske. Isotactics as a foundation for alignment and abstraction of behavioral models. In BPM, pages 335-351, 2012. doi:10.1007/978-3-642-32885-5_26.

[19] Michael O. Rabin and Dana Scott. Finite automata and their decision problems. IBM J. Res. Dev., 3(2):114-125, 1959. doi:10.1147/rd.32.0114

[20] Arend Rensink and Roberto Gorrieri. Vertical implementation. Inf. Comput., 170(1):95-133, 2001. doi:10.1006/inco. 2001.2967

[21] Michael Sipser. Introduction to the Theory of Computation. Thomson Course Technology, Boston, 2006.

[22] Arthur H. M. ter Hofstede, Wil M. P. van der Aalst, Michael Adams, and Nick Russell. Modern Business Process Automation: YAWL and Its Support Environment. Springer Publishing Company, Incorporated, 1 st edition, 2009.

[23] Wil M. P. van der Aalst. Inheritance of business processes: A journey visiting four notorious problems. In Petri Net Technology for Communication-Based Systems, pages 383-408, 2003. doi:10.1007/978-3-540-40022-6_19.

[24] Wil M. P. van der Aalst. Business alignment: using process mining as a tool for Delta analysis and conformance testing. Requir. Eng., 10(3):198-211, 2005. doi:10.1007/s00766-005-0001-x

[25] Wil M. P. van der Aalst, Arya Adriansyah, and Boudewijn F. van Dongen. Replaying history on process models for conformance checking and performance analysis. Wiley Interdiscip. Rev.-Data Mining Knowl. Discov., 2(2):182-192, 2012. doi: $10.1002 /$ widm.1045

[26] Rob J. van Glabbeek. The linear time-branching time spectrum (extended abstract). In CONCUR, volume 458 of Lecture Notes in Computer Science, pages 278-297. Springer, 1990. doi:10.1007/BFb0039066

[27] Rob J. van Glabbeek and Ursula Goltz. Well-behaved flow event structures for parallel composition and action refinement. Theor. Comput. Sci., 311(1-3):463-478, 2004. doi:10.1016/j.tcs.2003.10.031

[28] Barbara Weber, Manfred Reichert, and Stefanie Rinderle-Ma. Change patterns and change support features - enhancing flexibility in process-aware information systems. Data Knowl. Eng., 66(3):438-466, 2008. doi:10.1016/j.datak.2008.05. 001.

[29] Matthias Weidlich, Remco M. Dijkman, and Jan Mendling. The ICoP framework: Identification of correspondences between process models. In CAiSE, pages 483-498, 2010. doi:10.1007/978-3-642-13094-6_37

[30] Matthias Weidlich, Remco M. Dijkman, and Mathias Weske. Deciding behaviour compatibility of complex correspondences between process models. In BPM, pages 78-94, 2010. doi:10.1007/978-3-642-15618-2_8.

[31] Matthias Weidlich, Remco M. Dijkman, and Mathias Weske. Behaviour equivalence and compatibility of business process models with complex correspondences. Comput. J., 55(11):1398-1418, 2012. doi:10.1093/comjnl/bxs014

[32] F. Wijnhoven, T. Spil, R. Stegwee, and R.T.A. Fa. Post-merger IT integration strategies: An IT alignment perspective. J. Strateg. Inf. Syst., 15(1):5-28, 2006. doi:10.1016/j.jsis.2005.07.002

\section{Appendix A. Proofs}

Lemma 5.1. For $i \in\{1,2\}$, let $\Lambda_{i}$ be a set of labels and let $\Sigma_{i} \subseteq \Lambda_{i}^{*}$. Let $b$ be a bijection from $\Lambda_{1}$ to $\Lambda_{2}$, and

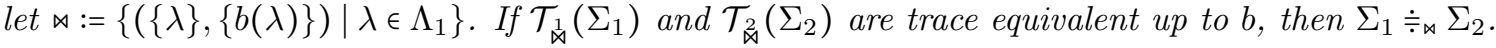

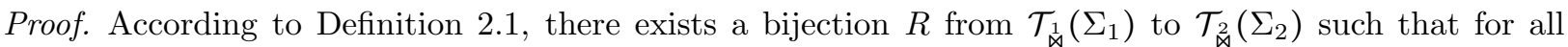
$\left(w_{1}, w_{2}\right) \in R$ it holds that (i) $\left|w_{1}\right|=\left|w_{2}\right|$, and (ii) for all $i \in\left\{1, \ldots,\left|w_{1}\right|\right\}$ it holds that $b\left(w_{1}(i)\right)=w_{2}(i)$. Let $\left(w_{1}, w_{2}\right) \in R$. Because $\bowtie \subseteq \wp_{=1}\left(\Lambda_{1}\right) \times \wp_{=1}\left(\Lambda_{2}\right)$, for all $i \in\left\{1, \ldots,\left|w_{1}\right|\right\}$ it holds that $\left|w_{1}(i)\right|=1=\left|w_{2}(i)\right|$. Therefore, for $i \in\{1,2\}, w_{i}$ is the only tactic of $w_{i}$. Note that for all $i \in\left\{1, \ldots,\left|w_{1}\right|\right\}$, it also holds that $w_{1}(i) \bowtie w_{2}(i)$ and, thus, $\left\{1, \ldots,\left|w_{1}\right|\right\} / w_{1}=\left\{1, \ldots,\left|w_{2}\right|\right\} / w_{2}$. Then, it trivially holds that $\left(w_{1}, w_{2}\right): w_{1} \bowtie w_{2}$, cf. Definition 3.6 one can use the identity relation on $\left\{1, \ldots,\left|w_{1}\right|\right\} / w_{1}$ as a bijection from $\left\{1, \ldots,\left|w_{1}\right|\right\} / w_{1}$ to $\left\{1, \ldots,\left|w_{2}\right|\right\} / w_{2}$ to justify this fact. Because $R$ is a bijection, we get $\Sigma_{1} \aleph_{\infty} \Sigma_{2}$. Because $R$ and $\bowtie$ are bijections, we get $\Sigma_{2} \aleph_{\aleph^{-1}} \Sigma_{1}$; note that $\bowtie$ is a bijection because $b$ is a bijection. Thus, it holds that $\Sigma_{1} \doteqdot_{\bowtie} \Sigma_{2}$.

Lemma 5.2. For $i \in\{1,2\}$, let $\Lambda_{i}$ be a set of labels and let $\Sigma_{i} \subseteq \Lambda_{i}^{*}$ be repetition-free. Let $b$ be a bijection from $\Lambda_{1}$

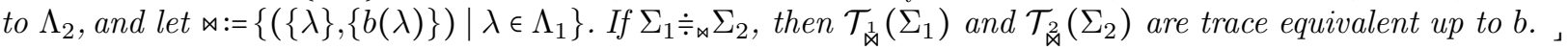

Proof. Because $\bowtie$ is a relation on singletons, for $i \in\{1,2\}, w_{i} \in \mathcal{T}_{\dot{\dot{\phi}}}\left(\Sigma_{i}\right)$ is the only tactic of $w_{i}$. Let $R:=\left\{(x, y) \in \mathcal{T}_{\triangleright}\left(\Sigma_{1}\right) \times \mathcal{T}_{\grave{\unrhd}}\left(\Sigma_{2}\right) \mid(x, y): x \bowtie y\right\}$. Let $\left(w_{1}, w_{2}\right) \in R$. Because $\Sigma_{1}$ and $\Sigma_{2}$ are repetition-free and because $\bowtie$ is a bijection, it holds that $w_{1}$ and $w_{2}$ are repetition-free; note that $\bowtie$ is a bijection because $b$ is a bijection. Therefore, for $i \in\{1,2\}$, it holds that $\left\{1, \ldots,\left|w_{i}\right|\right\} / w_{i}=\wp_{=1}\left(\left\{1, \ldots,\left|w_{i}\right|\right\}\right)$. Hence, $\left|w_{1}\right|=\left|w_{2}\right|$ and for all $k \in$ onetow $_{1}$ it holds that $w_{1}(k) \bowtie w_{2}(k)$ and, thus, $b\left(w_{1}(k)\right)=w_{2}(k)$. Next, we show that 
$R$ is a bijection from $\mathcal{T}_{\text {凶े }}\left(\Sigma_{1}\right)$ to $\mathcal{T}_{\grave{凶}}\left(\Sigma_{2}\right)$. Let $\left(w_{1}, w_{2}\right),\left(w_{1}^{\prime}, w_{2}^{\prime}\right) \in R$ and $i, j \in\{1,2\}, i \neq j$. Let us assume that $w_{i}=w_{i}^{\prime}$. As shown above, it holds that $\left|w_{i}\right|=\left|w_{j}\right|$ and $\left|w_{i}^{\prime}\right|=\left|w_{j}^{\prime}\right|$. Then, $w_{i}=w_{i}^{\prime}$ yields $\left|w_{j}\right|=\left|w_{j}^{\prime}\right|$. Additionally, for every $k \in\left\{1, \ldots,\left|w_{i}\right|\right\}$ it holds that $w_{1}(k) \bowtie w_{2}(k)$ and $w_{1}^{\prime}(k) \bowtie w_{2}^{\prime}(k)$. Because $w_{i}=w_{i}^{\prime}$ and $\bowtie$ is a bijection, $w_{j}(k)=w_{j}^{\prime}(k), k \in\left\{1, \ldots,\left|w_{j}\right|\right\}$. Hence, it holds that $w_{j}=w_{j}^{\prime}$. Finally, because $\Sigma_{1} \leqslant_{\infty} \Sigma_{2}$ and $\Sigma_{2} \aleph_{\aleph^{-1}} \Sigma_{1}$, for $i \in\{1,2\}$, it holds that for all $\sigma \in \Sigma_{i}$ there exists $\left(w_{1}, w_{2}\right) \in R$ such that $\mathcal{T}_{\dot{\dot{ }}}(\sigma)=w_{i}$. Thus, $R$

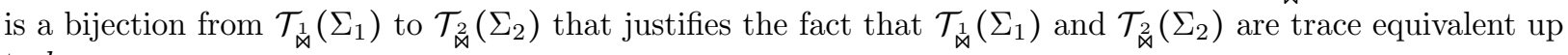
to $b$.

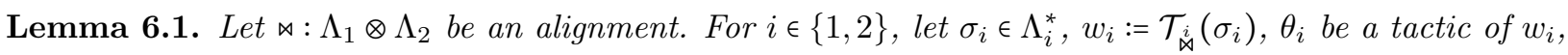
and let $\varphi_{i} \in \operatorname{LTL}[\dot{\dot{\varpi}}]$ be tactic-invariant w.r.t. $\Lambda_{i}$ and $\dot{\aleph}$. Let $\varphi_{1}$ and $\varphi_{2}$ be aligned by $\bowtie$, i.e., $\varphi_{1} \bowtie \varphi_{2}$, and let $\left(\theta_{1}, \theta_{2}\right): w_{1} \bowtie w_{2}$. Then, it holds that $w_{1} \vDash \varphi_{1}$ iff $w_{2} \vDash \varphi_{2}$.

Proof. For $i \in\{1,2\}$, it holds that $\theta_{i} \vDash \varphi_{i}$ iff $w_{i} \vDash \varphi_{i}$, because $\varphi_{i}$ is tactic-invariant. We show that $\theta_{1} \vDash \varphi_{1}$ iff $\theta_{2} \vDash \varphi_{2}$ by the structural induction on $\varphi_{1}$. Let $\varphi_{1}:=\bigvee_{K_{1} \in \mathcal{K}_{1}} K_{1}$ and $\varphi_{2}:=\bigvee_{K_{2} \in \mathcal{K}_{2}} K_{2}$, where $\mathcal{K}_{1} \subseteq \dot{\aleph}$ and $\mathcal{K}_{2} \subseteq \dot{\star}$ such that $\left(\mathcal{K}_{1} \times \grave{凶}\right) \cap \bowtie=\left(\bowtie \times \mathcal{K}_{2}\right) \cap \bowtie$. Then, for $j \in\{1,2\}, \theta_{j} \vDash \varphi_{j}$ iff $\theta_{j}(1) \cap \mathcal{K}_{j} \neq \varnothing$. Because $\theta_{1}(1) \bowtie \theta_{2}(1), \theta_{1}(1) \cap \mathcal{K}_{1} \neq \varnothing$ iff $\theta_{2}(1) \cap \mathcal{K}_{2} \neq \varnothing$ and, thus, it holds that $\theta_{1} \vDash \varphi_{1}$ iff $\theta_{2} \vDash \varphi_{2}$. Let $\varphi_{1}:=\neg \psi_{1}$ and $\varphi_{2}:=\neg \psi_{2}$ such that $\psi_{1} \bowtie \psi_{2}$. Then, for $k \in\{1,2\}$, it holds that $\theta_{k} \vDash \psi_{k}$ iff $\theta_{k} \not \varphi_{k}$. Using the inductive assumption, we conclude that $\theta_{1} \vDash \varphi_{1}$ iff $\theta_{2} \vDash \varphi_{2}$. Let $\varphi_{1}:=\psi_{1} \vee \psi_{1}^{\prime}$ and $\varphi_{2}:=\psi_{2} \vee \psi_{2}^{\prime}$ such that $\psi_{1} \bowtie \psi_{2}$ and $\psi_{1}^{\prime} \bowtie \psi_{2}^{\prime}$. Then, for $k \in\{1,2\}$, it holds that $\theta_{k} \vDash \varphi_{k}$ iff $\theta_{k} \vDash \psi_{k}$ or $\theta_{k} \vDash \psi_{k}^{\prime}$. Again, using the inductive assumption, we conclude that $\theta_{1} \vDash \varphi_{1}$ iff $\theta_{2} \vDash \varphi_{2}$. Let $\varphi_{1}:=\psi_{1} \cup \psi_{1}^{\prime}$ and $\varphi_{2}:=\psi_{2} \cup \psi_{2}^{\prime}$ such that $\psi_{1} \bowtie \psi_{2}$ and $\psi_{1}^{\prime} \bowtie \psi_{2}^{\prime}$. Then, for $k \in\{1,2\}$, it holds that $\theta_{k} \vDash \varphi_{k}$ iff there exists $\iota_{k} \in\left\{1, \ldots,\left|\theta_{k}\right|\right\}$ such that $\theta_{k}\left[\iota_{k}\right\rangle \vDash \psi_{k}^{\prime}$ and $\theta_{k}[\eta\rangle \vDash \psi_{k}$ for all $\eta \in\left\{1, \ldots, \iota_{k}-1\right\}$. By definition, for $k \in\{1,2\}$ and $\iota \in\left\{1, \ldots,\left|\theta_{k}\right|-1\right\}$ it holds that $\iota=\theta_{k} \iota+1$ implies $\theta_{k}(\iota)=\theta_{k}(\iota+1)$. Because of the stutter equivalence of LTL-formulae without the next operator [15, $\iota=\theta_{k} \iota+1$ also implies that $\theta_{k}[\iota\rangle$ and $\theta_{k}[\iota+1\rangle$ satisfy exactly the same LTL-formulae. Applying the inductive assumption then yields $\theta_{1} \vDash \varphi_{1}$ iff $\theta_{2} \vDash \varphi_{2}$. Finally, because $\psi_{1}$ and $\psi_{2}$ are tactic-invariant, it holds that $w_{1} \vDash \varphi_{1}$ iff $w_{2} \vDash \varphi_{2}$.

Lemma 6.2. $\hat{\Theta}=\hat{\Theta}^{\prime}$ iff $\varphi$ is tactic-invariant w.r.t. $\Lambda$ and $\kappa$.

Proof. We show both directions separately.

1. "1. $\Rightarrow 2$.": Let $w \in \mathcal{T}_{\kappa}\left(\Lambda^{*}\right)$ and $\theta \in \operatorname{Tactics}(w)$. Assume first $w \vDash \varphi$. Then, $w \in W$, and thus $\theta_{w} \in \hat{\Theta}$. Then, by assumption, $\theta \in \hat{\Theta}^{\prime}$. Hence, $\theta \in \Theta$. Therefore, $\theta \vDash \varphi$. Symmetrically, if $\theta \vDash \varphi$, then $\theta \in \Theta$, and thus also $\theta_{w} \in \hat{\Theta}^{\prime}$. Then, by assumption, $\theta_{w} \in \hat{\Theta}$. Then, $w \in W$, and therefore $w \vDash \varphi$.

2. "2. $\Rightarrow 1$.": Assume first $\theta_{w} \in \hat{\Theta}$. Then, $w \in \mathcal{T}_{\kappa}\left(\Lambda^{*}\right)$ with $w \vDash \varphi$, and $\theta \in \operatorname{Tactics}(w)$ of $w$. Because $\varphi$ is tactic-invariant, $\theta \vDash \varphi$. Thus, $\theta \in \Theta$, and $\theta_{w} \in \hat{\Theta}^{\prime}$. Now, assume $\theta_{w} \in \hat{\Theta}^{\prime}$. Then, there exists a tactic $\theta \in \Theta$ of some word $w$, such that $\theta \vDash \varphi$. Because $\varphi$ is tactic-invariant, $w \vDash \varphi$. Therefore, $\theta_{w} \in \hat{\Theta}^{\prime}$.

Lemma 6.3. It is decidable whether $\hat{\Theta}$ and $\hat{\Theta}^{\prime}$ are equal sets.

Proof. We construct an FSM $S_{\varphi}$ from $\varphi$, such that $\mathcal{L}\left(S_{\varphi}\right)$ is the set of all traces satisfying $\varphi$. Then, we construct FSMs $\underline{S}$ and $\bar{S}$ accepting all $w \in \mathcal{T}_{\kappa}\left(\Lambda^{*}\right)$ with $w(i) \neq \varnothing$ for all $i \in\{1, \ldots,|w|\}$, and $\theta \in \operatorname{Tactics}(w)$ and $\bar{S}$ accepts $w$, respectively. Let $S_{W}$ be the intersection of $S_{\varphi}$ and $\underline{S}$, and let $S_{\Theta}$ be the intersection of $S_{\varphi}$ and $\bar{S}$. We construct the FSM $\hat{S}_{\Theta}$ from $S_{W}$ by replacing each transition $q \stackrel{\mathcal{K}}{\rightarrow} S_{W} q^{\prime}$ by a transition $q \stackrel{(K, \mathcal{K})}{\longrightarrow} \hat{S}_{\Theta} q^{\prime}$ for each $K \in \mathcal{K}$. Then, we construct the FSM $\hat{S}_{\Theta}^{\prime}$ from $S_{\Theta}$ by replacing each transition $q \stackrel{\{K\}}{\longrightarrow} S_{\Theta} q^{\prime}$ by a transition $q \stackrel{(K, \mathcal{K})}{\longrightarrow} \hat{S}_{\Theta}, q^{\prime}$ for each $\mathcal{K} \in \mathcal{G}_{\kappa}(\Lambda)$ with $K \in \mathcal{K}$. Obviously, the languages of $\hat{S}_{\Theta}$ and $\hat{S}_{\Theta}^{\prime}$ are $\hat{\Theta}$ and $\hat{\Theta}^{\prime}$, respectively. Thus, we decide $\hat{\Theta}=\hat{\Theta}^{\prime}$ by deciding equivalence of $\hat{S}_{\Theta}$ and $\hat{S}_{\Theta}^{\prime}$.

Lemma 7.5. For $i \in\{1,2\}$, let $w_{i} \in \wp(\dot{i})^{*}$. Let $\left(w_{1}, w_{2}\right)$ be realisable in $\mathcal{W}\left(S_{1}, S_{2}, \bowtie\right)$ resulting in a node $v=\left(q_{1}, q_{2}, M\right)$. For each $\left(K_{1}, K_{2}\right) \in M$, there exist a tactic $\theta_{1}$ of $w_{1}$ and a tactic $\theta_{2}$ of $w_{2}$, such that $\left(\theta_{1}, \theta_{2}\right): w_{1} \bowtie w_{2}$ and for all $i \in\{1,2\}:\left|w_{i}\right|>0 \Rightarrow \theta_{i}\left(\left|w_{i}\right|\right)=\left\{K_{i}\right\}$. 
Proof by induction over the length of the realising path. Because $\left(w_{1}, w_{2}\right)$ is realisable, there exists a path $\pi$ of $\mathcal{W}\left(S_{1}, S_{2}, \bowtie\right)$ resulting in $v$, such that for $i \in\{1,2\}$, the restriction of $\pi[i]$ to non-empty sets yields $w_{i}$. Let $|\pi|=0$. Then, $w_{1}=w_{2}=\varepsilon$, trivially satisfying the requirements. Let $|\pi|=1$. Inspecting the rules $2(\mathrm{a})$ and $2(\mathrm{~b})$ of Definition 7.3, we find that only a) can produce an edge from the node $\left(q_{1}^{i n i}, q_{2}^{i n i}, \varnothing\right)$. Hence, for $i \in\{1,2\}$, $\pi[i]=\mathcal{K}_{i} \neq \varnothing$, and thus $w_{i}=\mathcal{K}_{i}$. Additionally, $M=\left(\mathcal{K}_{1} \times \mathcal{K}_{2}\right) \cap \bowtie$. Hence, for each $\left(G_{1}, G_{2}\right) \in M$, there also exists the tactics $\theta_{i}=\left\{G_{i}\right\}$ and $b$ proving $\left(\theta_{1}, \theta_{2}\right): w_{1} \bowtie w_{2}$. Now, let $|\pi|=n \geq 2$, and the proposition hold for all realising paths $\pi^{\prime}$ with $\left|\pi^{\prime}\right|<n$. Let the penultimate node of $\pi$ be $\hat{v}=\left(\hat{q}_{1}, \hat{q}_{2}, \hat{M}\right)$.

1. Assume that the last edge of $\pi$ is produced by rule $2(\mathrm{a})$ and labelled $\mathcal{K}_{1}, \mathcal{K}_{2}$. For $i \in\{1,2\}, w_{i}\left(\left|w_{i}\right|\right)=\mathcal{K}_{i}$, and, because $\hat{M} \neq \varnothing, M=\left\{\left(K_{1}, K_{2}\right) \in\left(\left(\mathcal{K}_{1} \times \mathcal{K}_{2}\right) \cap \bowtie\right) \backslash \hat{M} \mid \exists\left(\hat{K}_{1}, \hat{K}_{2}\right) \in \hat{M}: K_{1} \neq \hat{K}_{1} \wedge K_{2} \neq \hat{K}_{2}\right\} \neq \varnothing$. Let $\hat{w}_{i}=w_{i}(1) \ldots w_{i}\left(\left|w_{i}\right|-1\right)$. Let $\left(K_{1}, K_{2}\right) \in M$. Then, $\left(\hat{w}_{1}, \hat{w}_{2}\right)$ is realisable resulting in $\hat{v}$ by the prefix of $\pi$ with length $n-1>0$. Therefore, for each $\left(\hat{K}_{1}, \hat{K}_{2}\right) \in \hat{M}$, and $i \in\{1,2\}$, there exist a tactic $\hat{\theta}_{i}$ of $\hat{w}_{i}$ with $\hat{\theta}_{i}\left(\left|\hat{w}_{i}\right|\right)=\hat{K}_{i}$, and $\hat{b}$ proving $\left(\hat{\theta}_{1}, \hat{\theta}_{2}\right): \hat{w}_{1} \bowtie \hat{w}_{2}$. Then, for $i \in\{1,2\}, \hat{\theta}_{i}\left(\left|\hat{w}_{i}\right|\right) \neq\left\{K_{i}\right\}$. Now, set $\theta_{i}=\hat{\theta}_{i}\left\{K_{i}\right\}$. Then, $\left\{1, \ldots,\left|w_{i}\right|\right\} / \theta_{i}=\left\{1, \ldots, \hat{w}_{i}\right\} / \hat{\theta}_{i} \cup\left\{\left\{\left|w_{i}\right|\right\}\right\}$, that is, $\theta_{i}$ adds another equivalence class "to the end" of $\hat{\theta}_{i}$. Let $b=\hat{b} \cup\left\{\left(\left|w_{1}\right|,\left|w_{2}\right|\right)\right\}$. Because $b$ proves $\left(\hat{\theta}_{1}, \hat{\theta}_{2}\right): \hat{w}_{1} \bowtie \hat{w}_{2}$, and $K_{1} \bowtie K_{2}, \hat{b}$ proves $\left(\theta_{1}, \theta_{2}\right): w_{1} \bowtie w_{2}$.

2. Assume that the last edge of $\pi$ is produced by rule $2(\mathrm{~b})$ and labelled $\left(\mathcal{K}_{1}, \mathcal{K}_{2}\right)$. Then, there exist $i, j \in\{1,2\}, i \neq j, \lambda_{i} \in \Lambda_{i}, \mathcal{K}_{i}=\mathcal{G}_{\grave{\aleph}}\left(\lambda_{i}\right)$, such that $\hat{q}_{i}{\stackrel{\lambda_{i}}{\longrightarrow}}_{i} q_{i}$, and $M=\left\{\left(K_{1}, K_{2}\right) \in \hat{M} \mid \exists i \in\{1,2\}:\right.$ $\left.K_{i} \in \mathcal{K}_{i}\right\}$. Let $\hat{w}_{i}=w_{i}(1) \ldots w_{i}\left(\left|w_{i}\right|-1\right)$. Let $\hat{w}_{j}=w_{j}$. Then, $\left(\hat{w}_{1}, \hat{w}_{2}\right)$ is realisable resulting in $\hat{v}$ by the prefix of $\pi$ with length $n-1>0$. Therefore, for $k \in\{1,2\}$, and each $\left(K_{1}, K_{2}\right) \in \hat{M}$ (thus also $\left.\left(K_{1}, K_{2}\right) \in M\right)$, there exist a tactic $\hat{\theta}_{k}$ of $\hat{w}_{k}$ with $\hat{\theta}_{k}\left(\left|\hat{w}_{k}\right|\right)=K_{i}, \hat{\theta}_{j}\left(\left|\hat{w}_{j}\right|\right)=\hat{G}_{j}$, and $\hat{b}$ proving $\left(\hat{\theta}_{1}, \hat{\theta}_{2}\right): \hat{w}_{1} \bowtie \hat{w}_{2}$. Let $\theta_{i}=\hat{\theta}_{i}\left\{K_{i}\right\}, \hat{X}=\left\langle\left|\hat{w}_{i}\right|\right\rangle_{\hat{\theta}_{i}}$ be the "last" equivalence class in $\hat{\theta}_{i}$, and $X=\hat{X} \cup\left\{\left|w_{i}\right|\right\}$. Then, $\left\{1, \ldots,\left|w_{i}\right|\right\} / \theta_{i}=\left\{1, \ldots, \hat{w}_{i}\right\} / \hat{\theta}_{i} \backslash\{\hat{X}\} \cup\{X\}$, that is, the "last" equivalence class of $\theta_{i}$ is the union of the last equivalence class of $\hat{\theta}_{i}$ and $\left\{\left|w_{i}\right|\right\}$. Let $\theta_{j}=\hat{\theta}_{j}, \hat{Y}=\left\langle\left|\hat{w}_{j}\right|\right\rangle_{\hat{\theta}_{j}}$ and $b=\hat{b} \backslash\{(\hat{X}, \hat{Y})\} \cup\{(X, \hat{Y})\}$. Then, $b$ proves $\left(\theta_{1}, \theta_{2}\right): w_{1} \bowtie w_{2}$.

Lemma 7.6. For $i \in\{1,2\}$, let $\sigma_{i}$ be a prefix of some word in $\mathcal{L}\left(S_{i}\right)$, and $w_{i}=\mathcal{T}_{\dot{\downarrow}}\left(\sigma_{i}\right)$. Let for $i \in\{1,2\}, \theta_{i}$ be a tactic of $w_{i}$, such that $\left(\theta_{1}, \theta_{2}\right): w_{1} \bowtie w_{2}$. Then, $\left(w_{1}, w_{2}\right)$ is realisable resulting in some node $v=\left(q_{1}, q_{2}, M\right)$ with $\left|w_{1}\right|>0 \Rightarrow\left|w_{2}\right|=0 \wedge\left(\theta_{1}\left(\left|w_{1}\right|\right), \theta_{2}\left(\left|w_{2}\right|\right)\right) \in M$.

Proof by induction over the number of equivalence classes in the tactics. Let $w_{1}, w_{2}$ be traces with respective tactics $\theta_{1}, \theta_{2}$, such that $\left(\theta_{1}, \theta_{2}\right): w_{1} \bowtie w_{2}$. Let $n=\left|\{1, \ldots,|w|\}_{1} / \theta_{1}\right|$. We observe $n=\left|\left\{1, \ldots,\left|w_{2}\right|\right\} / \theta_{2}\right|$ because there exists a bijection $b$ aligning $\theta_{1}$ and $\theta_{2}$.

1. Let $n=0$. Then, $w_{1}=w_{2}=\varepsilon$. Then, the empty path proves realisability of $\left(w_{1}, w_{2}\right)$.

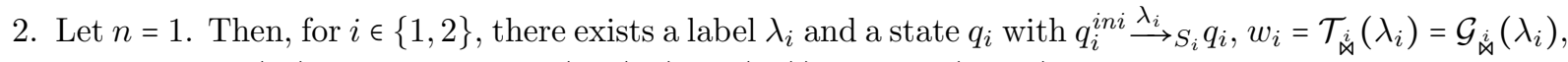

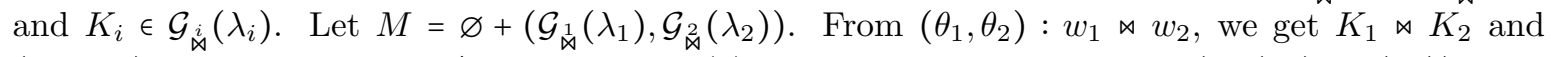
$\left(K_{1}, K_{2}\right) \in M$. Hence, $M \neq \varnothing$, and rule $2\left(\right.$ a) produces an edge labelled $\left(\mathcal{G}_{\bowtie}\left(\lambda_{1}\right), \mathcal{G}_{凶}\left(\lambda_{2}\right)\right)$ from $\left(q_{1}^{i n i}, q_{2}^{i n i}, \varnothing\right)$ to node $\left(q_{1}, q_{2}, M\right)$ in $\mathcal{W}\left(S_{1}, S_{2}, \bowtie\right)$.

3. Let $n>1$ and assume that the lemma holds for all alignable traces and respective tactics with $n-1$ equivalence classes. We first introduce an auxiliary notation for this part of the proof: Let $w$ be some trace with $|w|>1$ and $\theta$ be a tactic of $w$. Let $\operatorname{cut}(\theta)$ denote the maximal index in $\{1, \ldots,|w|\}$ with $\theta(i) \neq \theta(|w|)$. We observe $1 \leq \operatorname{cut}(\theta)<|w|$. Now, for $i \in\{1,2\}$, let $\hat{w}_{i}=w_{i}(1) \ldots w_{i}\left(\operatorname{cut}\left(\theta_{i}\right)\right)$ and $\hat{\theta}_{i}=\theta_{i}(1) \ldots \theta_{i}\left(\operatorname{cut}\left(\theta_{i}\right)\right)$. Let $\hat{b}=b \backslash\left\{\left(\left\langle\left|w_{1}\right|\right\rangle_{\theta_{1}},\left\langle\left|w_{2}\right|\right\rangle_{\theta_{2}}\right)\right\}$. Then, $\hat{b}$ proves $\left(\hat{\theta}_{1}, \hat{\theta}_{2}\right): \hat{w}_{1} \bowtie \hat{w}_{2}$, and we observe $\left|\left\{1, \ldots, \hat{w}_{i}\right\} / \theta_{i}\right|=n-1$ for $i \in\{1,2\}$. Let for $i \in\{1,2\}, \hat{\theta}_{i}\left(\operatorname{cut}\left(\theta_{i}\right)\right)=\left\{\hat{K}_{i}\right\}$. By assumption, $\left(\hat{w}_{1}, \hat{w}_{2}\right)$ is realisable resulting in a node $\left(\hat{q_{1}}, \hat{q_{2}}, \hat{M}\right)$ with $\left(\hat{K}_{1}, \hat{K}_{2}\right) \in \hat{M}$. By assumption, for $i \in\{1,2\}$, there exists a label $\lambda_{i}$, and a transition $\hat{q}_{i} \stackrel{\lambda_{i}}{\longrightarrow} q_{i}$ with $\mathcal{G}_{\grave{\downarrow}}\left(\lambda_{i}\right)=w_{i}\left(\operatorname{cut}\left(\theta_{i}\right)+1\right)$ and

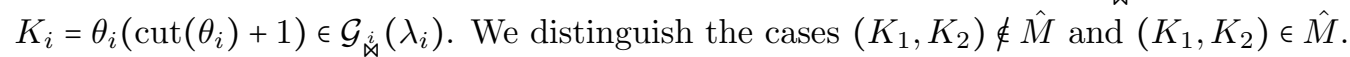


(a) Let $\left(K_{1}, K_{2}\right) \notin \hat{M}$. Let $M=\hat{M}+\left(\mathcal{G}_{\text {令 }}\left(\lambda_{1}\right), \mathcal{G}_{\text {岗 }}\left(\lambda_{2}\right)\right)$. From $K_{1} \bowtie K_{2},\left(\hat{K}_{1}, \hat{K}_{2}\right) \in \hat{M}, \hat{K}_{1} \neq K_{1}$ and $\hat{K}_{2} \neq K_{2}$, we conclude $\left(K_{1}, K_{2}\right) \in M$ and $M \neq \varnothing$. Therefore, rule 2(a) produces an edge labelled

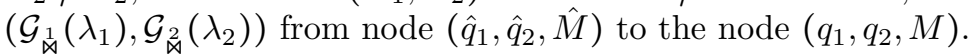

(b) Let $\left(K_{1}, K_{2}\right) \in \hat{M}$. Let $M^{\prime}=\hat{M}+\left(\mathcal{G}_{\text {ฝ }}\left(\lambda_{1}\right), \varnothing\right)$ and $M=M^{\prime}+\left(\varnothing, \mathcal{G}_{\grave{\aleph}}\left(\lambda_{2}\right)\right)$. Then, $\left(K_{1}, K_{2}\right) \in M^{\prime} \cap M$, and hence $M^{\prime} \neq \varnothing \neq M$. From $M^{\prime} \neq \varnothing$ and $M \neq \varnothing$, we conclude that rule 2(b) produces an edge

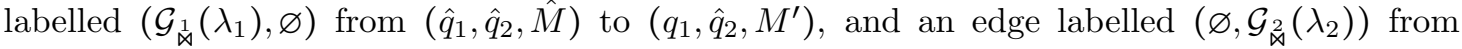
$\left(q_{1}, \hat{q}_{2}, M^{\prime}\right)$ to $\left(q_{1}, q_{2}, M\right)$.

If for $i \in\{1,2\}$, $\operatorname{cut}\left(\theta_{i}\right)=\left|w_{i}\right|-1$, we are finished. Otherwise, rule 2(b) produces the remaining edges: We first add the edges for the remaining elements of $w_{1}$, and then for the remaining elements of $w_{2}$, resulting in edges labelled

$$
\left(w_{1}\left(\operatorname{cut}\left(\theta_{i}\right)+2\right), \varnothing\right) \ldots\left(w_{1}\left(\left|w_{i}\right|\right), \varnothing\right)\left(\varnothing, w_{2}\left(\operatorname{cut}\left(\theta_{i}\right)+2\right)\right) \ldots\left(\varnothing, w_{2}\left(\left|w_{2}\right|\right)\right)
$$

and nodes $\left(q_{1}^{1}, q_{2}, M_{1}\right) \ldots\left(q_{1}^{\ell}, q_{2}, M_{\ell}\right)\left(q_{1}^{\ell}, q_{2}^{1}, M_{\ell+1}\right) \ldots\left(q_{1}^{\ell}, q_{2}^{m}, M_{\ell+m}\right)$, where for each $1 \leq j \leq \ell+m$, we exploit $\left(K_{1}, K_{2}\right) \in M_{j}$.

Lemma 7.8. The following statements are equivalent:

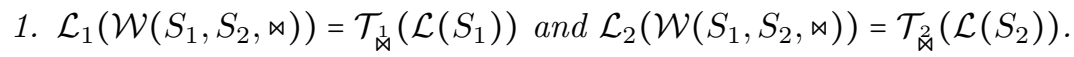

2. $\mathcal{L}\left(S_{1}\right) \doteqdot_{\bowtie} \mathcal{L}\left(S_{2}\right)$.

Proof. We show both directions separately.

1. " $1 \Rightarrow 2$ ": We show: For each $w_{1} \in \mathcal{T}_{\grave{\aleph}}\left(\mathcal{L}\left(S_{1}\right)\right)$, there exists $w_{2} \in \mathcal{T}_{2}\left(\mathcal{L}\left(S_{2}\right)\right)$, and tactics $\theta_{1}$ and $\theta_{2}$ of $w_{1}$ and $w_{2}$, respectively, such that there is $b$ proving $\left(\theta_{1}, \theta_{2}\right): w_{1} \bowtie w_{2}$. From $w_{1} \in \mathcal{T}_{\triangleright}\left(\mathcal{L}\left(S_{1}\right)\right)$ and the assumption, we get $w_{1} \in \mathcal{L}_{1}\left(\mathcal{W}\left(S_{1}, S_{2}, \bowtie\right)\right)$. Hence, there exists a path $\pi$ of $\mathcal{W}\left(S_{1}, S_{2}, \bowtie\right)$, such that $\pi[i]_{\neq \varnothing}=w_{1}$, resulting in a node $\left(q_{1}, q_{2}, M\right)$ satisfying $q_{1} \in F_{1}$ and $q_{2} \in F_{2}$. Let $w_{2}=\pi[2]_{\neq \varnothing}$. Then, by definition, $\left(w_{1}, w_{2}\right)$ are realisable. Applying Lemma 7.5 yields the existence of required $\theta_{1}, \theta_{2}$ and $b$. It now remains to be shown that $w_{2} \in \mathcal{T}_{\grave{\unrhd}}\left(\mathcal{L}\left(S_{2}\right)\right)$, which follows directly from the assumption.

2. " $2 \Rightarrow 1 "$ :

- We show $\mathcal{L}_{1}\left(\mathcal{W}\left(S_{1}, S_{2}, \bowtie\right)\right) \subseteq \mathcal{T}_{\text {\} }\left(\mathcal{L}\left(S_{1}\right)\right) \text {. Let } w_{1} \in \mathcal{L}_{1}\left(\mathcal{W}\left(S_{1}, S_{2}, \bowtie\right)\right) \text {. Hence, there exists a path }$ $\pi$ of $\mathcal{W}\left(S_{1}, S_{2}, \bowtie\right)$, such that $\pi[1]_{\neq \varnothing}=w_{1}$, resulting in a node $\left(q_{1}, q_{2}, M\right)$ satisfying $q_{1} \in F_{1}$ and $q_{2} \in F_{2}$. Now, we can show by induction, that there exists an accepting path of $S_{1}$ accepting some $\sigma_{1}$ with $\mathcal{T}_{\text {ऐ }}\left(\sigma_{1}\right)=w_{1}$.

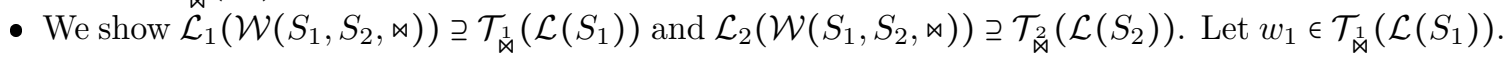
Then, by assumption, there exists $w_{2} \in \mathcal{T}_{\grave{\aleph}}\left(\mathcal{L}\left(S_{2}\right)\right), \theta_{1}, \theta_{2}$ and $b$ proving $\left(\theta_{1}, \theta_{2}\right): w_{1} \bowtie w_{2}$. Hence, by Lemma 7.6. $\left(w_{1}, w_{2}\right)$ is realisable, yielding some node $\left(q_{1}, q_{2}, M\right)$. Hence, there exists a path

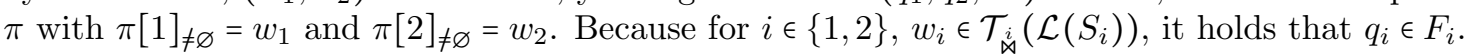
Hence, for $i \in\{1,2\}, w_{i} \in \mathcal{L}_{i}\left(\mathcal{W}\left(S_{1}, S_{2}, \bowtie\right)\right)$.

Lemma 7.9. For $i \in\{1,2\}$, the following problem is decidable:

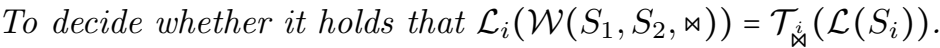

Proof. $\mathcal{W}\left(S_{1}, S_{2}, \bowtie\right)$ is obviously finite and computable. Further, one can construct an FSM $S$ accepting $\mathcal{L}_{i}\left(\mathcal{W}\left(S_{1}, S_{2}, \bowtie\right)\right), i \in\{1,2\}$, by following this procedure:

1. Each node of $\mathcal{W}\left(S_{1}, S_{2}, \bowtie\right)$ is a state of $S$.

2. For each edge $\left(v, \mathcal{K}_{1}, \mathcal{K}_{2}, v^{\prime}\right)$ of $\mathcal{W}\left(S_{1}, S_{2}, \bowtie\right)$ introduce a transition $v \stackrel{K_{i}}{\longrightarrow} S v^{\prime}$,

3. Set the node $\left(q_{1}^{\text {ini }}, q_{2}^{\text {ini }}, \varnothing\right)$ as the initial state.

4. A state $\left(q_{1}, q_{2}, M\right)$ is a final state of $S$ iff $q_{1} \in F_{1}$ and $q_{2} \in F_{2}$.

5. Finally, remove all $\varnothing$-transitions with a standard $\varepsilon$-removal algorithm [21].

We decide $\mathcal{L}_{i}\left(\mathcal{W}\left(S_{1}, S_{2}, \bowtie\right)\right)=\mathcal{T}_{\dot{\dot{\downarrow}}}\left(\mathcal{L}\left(S_{i}\right)\right)$ by checking language equivalence of $S$ and $S_{i}$. 


\section{Appendix B. Example for Lemma 6.3}

This section introduces an example for the decision procedure explained in the proof of Lemma 6.3. We consider the example alignment $\bowtie$ given by Figure 1, and the $\operatorname{LTL}[\stackrel{\aleph}{\bowtie}]$-formula $\varphi=\{t, u\} \cup(\{s, v\} \vee\{s, w, x\})$. Then, $S_{\varphi}$ consists of an initial state $q_{1}$, a final state $q_{2}$, for each $\mathcal{K} \subseteq \grave{\otimes}$ a transition $q_{2} \stackrel{\mathcal{K}}{\rightarrow} q_{2}$ (in the following abbreviated as $q_{2} \stackrel{*}{\rightarrow} q_{2}$ ), and for each $\mathcal{K} \subseteq \underset{\otimes}{\otimes}$ satisfying $\{s, v\} \in \mathcal{K}$ implies $\{s, w, x\} \notin \mathcal{K}$ a transition $q_{1} \stackrel{\mathcal{K}}{\rightarrow} q_{2}$, namely:

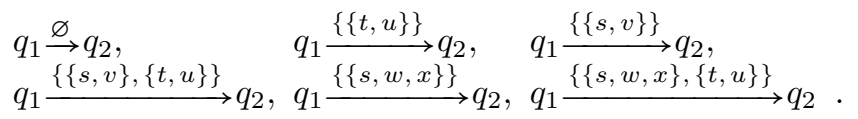

$S_{W}$ has the same initial and final states, and the following transitions:

$$
\begin{aligned}
& q_{1} \stackrel{\{\{t, u\}\}}{\longrightarrow} q_{2}, q_{1} \stackrel{\{\{s, v\}\}}{\longrightarrow} q_{2}, q_{1} \stackrel{\{\{s, w, x\}\}}{\longrightarrow} q_{2}, \\
& q_{2} \stackrel{\{\{t, u\}\}}{\longrightarrow} q_{2}, q_{2} \stackrel{\{\{s, v\}\}}{\longrightarrow} q_{2}, q_{2} \stackrel{\{\{s, w, x\}\}}{\longrightarrow} q_{2}, q_{2} \stackrel{\{\{s, v\},\{s, w, x\}\}}{\longrightarrow} q_{2} .
\end{aligned}
$$

$S_{\Theta}$ has the same initial and final states, and the following transitions:

$$
\begin{aligned}
& q_{1} \stackrel{\{\{t, u\}\}}{\longrightarrow} q_{2}, q_{1} \stackrel{\{\{s, v\}\}}{\longrightarrow} q_{2}, q_{1} \stackrel{\{\{s, w, x\}\}}{\longrightarrow} q_{2}, \\
& q_{2} \stackrel{\{\{t, u\}\}}{\longrightarrow} q_{2}, q_{2} \stackrel{\{\{s, v\}\}}{\longrightarrow} q_{2}, q_{2} \stackrel{\{\{s, w, x\}\}}{\longrightarrow} q_{2} .
\end{aligned}
$$

$\hat{S}_{\Theta}$ has the initial and final states, and the following transitions:

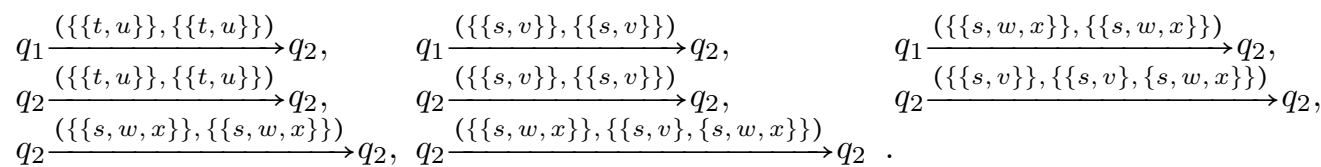

$\hat{S}_{\Theta}^{\prime}$ evolves from $\hat{S}_{\Theta}$ by adding the following transitions:

$$
q_{1} \stackrel{(\{\{s, v\}\},\{\{s, v\},\{s, w, x\}\})}{\longrightarrow} q_{2}, q_{1} \stackrel{(\{\{s, w, x\}\},\{\{s, v\},\{s, w, x\}\})}{\longrightarrow} q_{2} .
$$

It is obvious that $\hat{S}_{\Theta}^{\prime}$ accepts the language of $\hat{S}_{\Theta}$, and, in addition, all those sequences that start with $(\{\{s, v\}\},\{\{s, v\},\{s, w, x\}\})$ and $(\{\{s, w, x\}\},\{\{s, v\},\{s, w, x\}\})$, respectively. Therefore, $\varphi$ is not tacticinvariant. 